\title{
Calibration of the Super-Kamiokande Detector
}

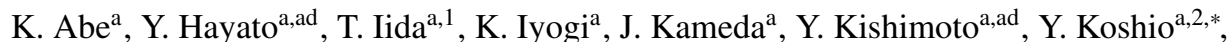
Ll. Marti ${ }^{\mathrm{a}, 3}$, M. Miura ${ }^{\mathrm{a}}$, S. Moriyama ${ }^{\mathrm{a}, \mathrm{ad}}$, M. Nakahata ${ }^{\mathrm{a}, \mathrm{ad}}$, Y. Nakano $^{\mathrm{a}}$, S. Nakayama ${ }^{\mathrm{a}}$, Y. Obayashi ${ }^{\mathrm{a}, 4}$, H. Sekiya ${ }^{\mathrm{a}}$, M. Shiozawa ${ }^{\mathrm{a}, \mathrm{ad}}$, Y. Suzuki $^{\mathrm{a}, \mathrm{ad}}$, A. Takeda ${ }^{\mathrm{a}}$, Y. Takenaga ${ }^{\mathrm{a}}$,

H. Tanaka ${ }^{\mathrm{a}}$, T. Tomura ${ }^{\mathrm{a}}$, K. Ueno ${ }^{\mathrm{a}, 5}$, R. A. Wendell ${ }^{\mathrm{a}}$, T. Yokozawa ${ }^{\mathrm{a}, 6}$, T. J. Irvine ${ }^{\mathrm{b}}$, H. Kaji ${ }^{\mathrm{b}}$,

T. Kajita ${ }^{\text {,ad }}$, K. Kaneyuki ${ }^{\text {b,ad,12, }}$, K. P. Lee ${ }^{\mathrm{b}}$, Y. Nishimura ${ }^{\mathrm{b}}$, K. Okumura ${ }^{\mathrm{b}}$, T. McLachlan ${ }^{\mathrm{b}}$, L. Labarga ${ }^{\text {c }}$, E. Kearns ${ }^{\text {e,ad }}$, J. L. Raaf ${ }^{\text {e }}$ J. L. Stone ${ }^{\text {e,ad }}$, L. R. Sulak ${ }^{\text {e }}$ S. Berkman ${ }^{\text {d }}$, H. A. Tanaka ${ }^{\text {, }}$ S. Tobayama ${ }^{\mathrm{d}}$, M. Goldhaber ${ }^{\mathrm{f}}, 12$, K. Bays ${ }^{\mathrm{g}}$, G. Carminati ${ }^{\mathrm{g}}$, W. R. Kropp ${ }^{\mathrm{g}}$, S. Mine ${ }^{\mathrm{g}}$,

A. Renshaw ${ }^{g}$, M. B. Smy ${ }^{\text {g }}$, H. W. Sobel ${ }^{\text {g,ad }}$, K. S. Ganezer ${ }^{\text {h }}$, J. Hill ${ }^{\text {h }}$, W. E. Keig ${ }^{\text {, J. S. Jang }}$, , $^{\text {, }}$

J. Y. Kim ${ }^{i}$, I. T. Lim ${ }^{i}$, N. Hong ${ }^{i}$, T. Akiri ${ }^{j}$, J. B. Albert ${ }^{j}$, A. Himmel ${ }^{j}$, K. Scholberg ${ }^{j}$,ad,

C. W. Walter ${ }^{\mathrm{j}, \text { ad }}$, T. Wongjirad ${ }^{\mathrm{j}}$, T. Ishizuka ${ }^{\mathrm{k}}, \mathrm{S}_{\text {. Tasaka }}{ }^{\mathrm{l}}$, J. G. Learned ${ }^{\mathrm{m}}$, S. Matsuno $^{\mathrm{m}}$,

S. N. Smith ${ }^{\mathrm{m}}$, T. Hasegawa ${ }^{\mathrm{n}}$, T. Ishida ${ }^{\mathrm{n}}$, T. Ishii ${ }^{\mathrm{n}}$, T. Kobayashi ${ }^{\mathrm{n}}$, T. Nakadaira ${ }^{\mathrm{n}}$,

K. Nakamura ${ }^{\mathrm{n}, \mathrm{ad}}$, K. Nishikawa ${ }^{\mathrm{n}}$, Y. Oyama ${ }^{\mathrm{n}}$, K. Sakashita ${ }^{\mathrm{n}}$, T. Sekiguchi ${ }^{\mathrm{n}}$, T. Tsukamoto ${ }^{\mathrm{n}}$,

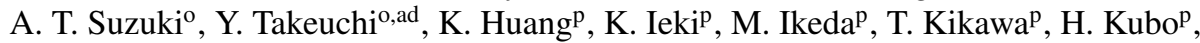

A. Minamino ${ }^{\mathrm{p}}$, A. Murakami ${ }^{\mathrm{p}}$, T. Nakaya ${ }^{\mathrm{p}, \mathrm{ad}}$, M. Otani $^{\mathrm{p}, 8}$, K. Suzuki $^{\mathrm{p}}$, S. Takahashi $^{\mathrm{p}}$,

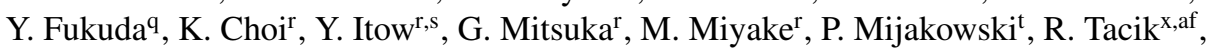

J. Hignight ${ }^{\mathrm{u}}$, J. Imber ${ }^{\mathrm{u}}$, C. K. Jung ${ }^{\mathrm{u}}$, I. Taylor ${ }^{\mathrm{u}}$, C. Yanagisawa ${ }^{\mathrm{u}}$, Y. Idehara ${ }^{\mathrm{v}, 9}$, H. Ishino ${ }^{\mathrm{v}}$,

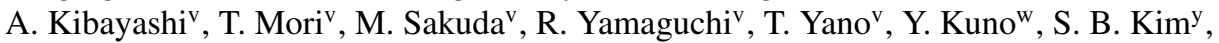

B. S. Yang ${ }^{\mathrm{y}, 10}$, H. Okazawa ${ }^{\mathrm{z}}$, Y. Choi ${ }^{\text {aa }}$, K. Nishijima ${ }^{\text {ab }}$, M. Koshiba ${ }^{\text {ac }}$, Y. Totsuka ${ }^{\text {ac, }}{ }^{12}$,

M. Yokoyama ${ }^{\text {ac,ad }}$, K. Martens ${ }^{\text {ad }}$, M. R. Vagins ${ }^{\text {ad, }}$, J. F. Martin ${ }^{\text {ae }}$, P. de Perio ${ }^{\text {ae }}$, A. Konaka ${ }^{\text {af }}$, M. J. Wilking ${ }^{\text {af }}$, S. Chen ${ }^{\text {ag }}$, Y. Heng ${ }^{\text {ag, } 11}$, H. Sui ${ }^{\text {ag }}$, Z. Yang ${ }^{\text {ag }}$, H. Zhang ${ }^{\text {ag }}$, Y. Zhenwei ${ }^{\text {ag }}$, K. Connolly ${ }^{\text {ah }}$, M. Dziomba ${ }^{\text {ah }}$, R. J. Wilkes ${ }^{\text {ah }}$

\footnotetext{
${ }^{a}$ Kamioka Observatory, Institute for Cosmic Ray Research, University of Tokyo, Kamioka, Gifu 506-1205, Japan

${ }^{b}$ Research Center for Cosmic Neutrinos, Institute for Cosmic Ray Research, University of Tokyo, Kashiwa, Chiba 277-8582, Japan

${ }^{c}$ Department of Theoretical Physics, University Autonoma Madrid, 28049 Madrid, Spain

${ }^{d}$ Department of Physics and Astronomy, University of British Columbia, Vancouver, B.C., V6T1Z4, Canada ${ }^{e}$ Department of Physics, Boston University, Boston, MA 02215, USA

${ }^{f}$ Physics Department, Brookhaven National Laboratory, Upton, NY 11973, USA

${ }^{g}$ Department of Physics and Astronomy, University of California, Irvine, Irvine, CA 92697-4575, USA

${ }^{h}$ Department of Physics, California State University, Dominguez Hills, Carson, CA 90747, USA

${ }^{i}$ Department of Physics, Chonnam National University, Kwangju 500-757, Korea ${ }^{j}$ Department of Physics, Duke University, Durham NC 27708, USA

${ }^{k}$ Junior College, Fukuoka Institute of Technology, Fukuoka, Fukuoka 811-0295, Japan

${ }^{l}$ Department of Physics, Gifu University, Gifu, Gifu 501-1193, Japan

${ }^{m}$ Department of Physics and Astronomy, University of Hawaii, Honolulu, HI 96822, USA

${ }^{n}$ High Energy Accelerator Research Organization (KEK), Tsukuba, Ibaraki 305-0801, Japan ${ }^{\circ}$ Department of Physics, Kobe University, Kobe, Hyogo 657-8501, Japan

${ }^{p}$ Department of Physics, Kyoto University, Kyoto, Kyoto 606-8502, Japan

${ }^{q}$ Department of Physics, Miyagi University of Education, Sendai, Miyagi 980-0845, Japan

${ }^{r}$ Solar Terrestrial Environment Laboratory, Nagoya University, Nagoya, Aichi 464-8602, Japan

${ }^{s}$ Kobayashi-Maskawa Institute for the Origin of Particles and the Universe, Nagoya University, Nagoya, Aichi 464-8602, Japan

${ }^{t}$ National Centre For Nuclear Research, 00-681 Warsaw, Poland

${ }^{u}$ Department of Physics and Astronomy, State University of New York, Stony Brook, NY 11794-3800, USA

${ }^{v}$ Department of Physics, Okayama University, Okayama, Okayama 700-8530, Japan

${ }^{w}$ Department of Physics, Osaka University, Toyonaka, Osaka 560-0043, Japan

${ }^{x}$ Department of Physics, University of Regina, 3737 Wascana Parkway, Regina, SK, S4S OA2, Canada ${ }^{y}$ Department of Physics, Seoul National University, Seoul 151-742, Korea

${ }^{z}$ Department of Informatics in Social Welfare, Shizuoka University of Welfare, Yaizu, Shizuoka, 425-8611, Japan
} 
${ }^{a b}$ Department of Physics, Tokai University, Hiratsuka, Kanagawa 259-1292, Japan

${ }^{a c}$ The University of Tokyo, Bunkyo, Tokyo 113-0033, Japan

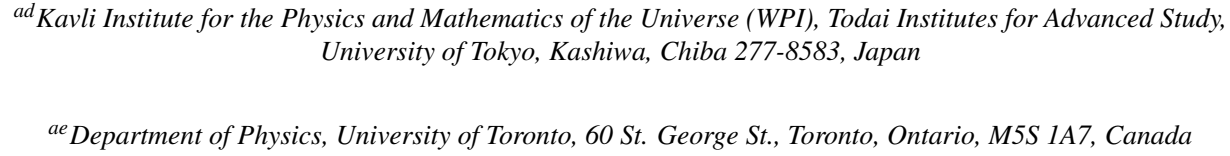

\begin{abstract}
Procedures and results on hardware-level detector calibration in Super-Kamiokande (SK) are presented in this paper. In particular, we report improvements made in our calibration methods for the experimental phase IV in which new readout electronics have been operating since 2008 .

The topics are separated into two parts. The first part describes the determination of constants needed to interpret the digitized output of our electronics so that we can obtain physical numbers such as photon counts and their arrival times for each photomultiplier tube (PMT). In this context, we developed an in-situ procedure to determine high-voltage settings for PMTs in large detectors like SK, as well as a new method for measuring PMT quantum efficiency and gain in such a detector.
\end{abstract}

The second part describes modeling of the detector in Monte Carlo simulations, including, in particular, the optical properties of the water target and their variability over time. Detailed studies on water quality are also presented.

As a result of this work, we have achieved a precision sufficient for physics analyses over a wide energy range (from a few $\mathrm{MeV}$ to above $1 \mathrm{TeV}$ ). For example, charge determination was at the level of $1 \%$, and the timing resolution was $2.1 \mathrm{~ns}$ at the one-photoelectron charge level and $0.5 \mathrm{~ns}$ at the 100-photoelectron charge level.

Keywords: Neutrino detector, Detector calibration 


\section{Overview}

The Super-Kamiokande (SK) is an imaging water Cherenkov detector used to study neutrinos from various sources (the Sun, the atmosphere, past supernovae, and an accelerator) and to search for proton decay. Since the beginning of the experiment, various calibration procedures have been employed to determine the properties of the detector. Calibrations carried out in the initial phases of SK were reported in [1]. This paper discusses recent improvements and adaptions to our calibration methods.

In Section 2, we provide an overview of the SK detector with emphasis on recent electronics upgrade. Calibration of the inner detector is described in Section 3 Section 3.1 describes basic calibration of photomultiplier tubes (PMTs). Section 3.2 examines optical photon propagation in the detector. Section 4 describes calibration of the outer detector.

Some specialized calibration procedures are reported elsewhere; use of an electron accelerator [2] and ${ }^{16} \mathrm{~N}$ source [3] for solar and supernova neutrino analyses [4]. Use of cosmic ray muons, $\pi^{0}$ generated in the detector, and muon decay electrons for atmospheric and accelerator neutrino oscillation, and proton decay analyses are described in [5, 6].

\section{Super-Kamiokande detector}

\subsection{Introduction}

The SK detector is a cylindrical tank (39.3 m diameter and $41.4 \mathrm{~m}$ height) filled with 50 kilotons of ultra-pure water. It is located $1000 \mathrm{~m}$ underground (2,700 $\mathrm{m}$ water equivalent) in the Kamioka mine in Gifu Prefecture, Japan. Inside its stainless steel tank, a stainless steel structure supports the PMTs and optically separates the tank into inner (ID) and outer detector (OD) [1]. The optical isolation of ID and OD is effected by black polyethylene terephthalate sheets ("black sheets") on the inside of the barrier and highly reflective Tyvek sheets on its outside. Tank walls are also lined with Tyvek sheets. The experiment began data-taking in April 1996 and was shut down for maintenance in July 2001, this initial phase is called "SK-I". Because of an accident during the ensuing upgrade work, the experiment resumed in October 2002

\footnotetext{
*Corresponding author. Tel.: +81 862517817 ; fax: +81 862517830 .

Email address: koshio@fphy.hep.okayama-u.ac.jp (Y. Koshio)

${ }^{1}$ Present address: Research Center for Nuclear Physics, Osaka University, 10-1 Mihogaoka, Ibaraki, Osaka, $567-$ 0047, Japan

${ }^{2}$ Present address: Department of Physics, Okayama University, Okayama, Okayama 700-8530, Japan

${ }^{3}$ Present address: Kamioka Satellite, Kavli Institute for the Physics and Mathematics of the Universe (WPI), Todai Institutes for Advanced Study, University of Tokyo, Kamioka, Gifu 506-1205, Japan

${ }^{4}$ Present address: Kavli Institute for the Physics and Mathematics of the Universe (WPI), Todai Institutes for Advanced Study, University of Tokyo, Kashiwa, Chiba 277-8583, Japan

${ }^{5}$ Present address: Department of Earth and Space Science, Osaka University, Toyonaka, Osaka, 560-0043, Japan

${ }^{6}$ Present address: Department of Physics, Osaka City University, Sugimoto 3-3-138, Sumiyoshi-ku, Osaka, 558-8585, Japan

${ }^{7}$ Present address: GIST College, Gwangju Institute of Science and Technology, Gwangju 500-712, Korea

${ }^{8}$ Present address: Research Center for Neutrino Science, Tohoku University, Sendai 980-8578, Japan

${ }^{9}$ Present address: Shizuoka Plant, Koito manufacturing CO., LTD., 500, Kitawaki, Shimizu-ku, Shizuoka 424-8764, Japan

${ }^{10}$ Present address: Kamioka Observatory, Institute for Cosmic Ray Research, University of Tokyo, Kamioka, Gifu 506-1205, Japan

${ }^{11}$ Present address: CERN PH-Division, Bat 32, R-A-18, CH-1211, Geneva, Switzerland

${ }^{12}$ Deceased.
} 
with only about half of its original number of ID-PMTs. To prevent further accidents from that time on, all ID-PMTs were encased in fiber-reinforced plastic (FRP) cases with acrylic front windows. The phase from October 2002 until another shutdown for full reconstruction, which started in October 2005, is called "SK-II". In July 2006, the experiment resumed with full number of PMTs, it stopped briefly for an electronics upgrade in September of 2008. That phase is called "SK-III". The period of data-taking after the electronics upgrade, which started after September 2008, is called "SK-IV", that period is ongoing as of the time of writing this paper. The photodetector coverage differed in most phases. The SK phases and their main characteristics are summarized in Table 1] A detailed description of the SK-I detector was published [1], where detector calibrations were also discussed. Since then, there have been major improvements to the calibrations, especially those related to the optimization of high-voltage settings for each PMT, in understanding of the PMT-to-PMT variations in quantum efficiency and gain, and in understanding of optical properties of water in the detector. These improvements are described in this paper.

\begin{tabular}{|c|c||c|c|c|c|}
\hline \multicolumn{2}{|c||}{ Phase } & SK-I & SK-II & SK-III & SK-IV \\
\hline Period & $\begin{array}{c}\text { start } \\
\text { end }\end{array}$ & $\begin{array}{c}\text { 1996 Apr. } \\
\text { 2001 Jul. }\end{array}$ & $\begin{array}{c}\text { 2002 Oct. } \\
\text { 2005 Oct. }\end{array}$ & $\begin{array}{c}\text { 2006 Jul. } \\
\text { 2008 Sep. }\end{array}$ & $\begin{array}{c}\text { 2008 Sep. } \\
\text { (running) }\end{array}$ \\
\hline $\begin{array}{c}\text { Number } \\
\text { of } \\
\text { PMTs }\end{array}$ & ID & $\begin{array}{c}11146 \\
(40 \%)\end{array}$ & $\begin{array}{c}5182 \\
(19 \%)\end{array}$ & $\begin{array}{c}11129 \\
(40 \%)\end{array}$ & $\begin{array}{c}11129 \\
(40 \%)\end{array}$ \\
\cline { 2 - 6 } & OD & no & yes & yes & yes \\
\hline $\begin{array}{c}\text { Anti-implosion } \\
\text { container }\end{array}$ & no & no & yes & yes \\
\hline $\begin{array}{c}\text { OD segmentation } \\
\text { (Figure 33) }\end{array}$ & \multicolumn{3}{|c|}{$\begin{array}{c}\text { ATM (ID) } \\
\text { OD QTC[1] (OD) }\end{array}$} & QBEE \\
\hline $\begin{array}{c}\text { Front-end } \\
\text { electronics }\end{array}$ & \multicolumn{5}{c}{} \\
\hline
\end{tabular}

Table 1: Detector configuration for the SK experiment phases up to the current time. The values in parentheses below the number of PMTs in the ID show percent photo-coverage of the surface.

\subsection{SK-IV electronics upgrade}

In phases SK-I, SK-II, and SK-III, signals from the ID-PMTs were processed by custom electronics modules called analog timing modules (ATMs) [8]. These modules contained both charge-to-analog converters (QAC) and time-to-analog converters (TAC). The dynamic range was from $0 \sim 450$ pico Coulomb (pC) with $0.2 \mathrm{pC}$ resolution for charge and from $-300 \sim 1000 \mathrm{~ns}$ with 0.4 ns resolution for time. There were two pairs of QAC/TAC for each PMT input signal, this prevented dead time and allowed the readout of multiple sequential hits that may arise, e.g. from electrons that are decay products of stopping muons.

To ensure stable data-taking over the next decade and to improve the throughput of data acquisition systems, the SK electronics system was upgraded in September 2008. For SK-IV, new front-end electronics modules, called QTC-based electronics with Ethernet (QBEE) [19], were developed and installed for measuring arrival times and integrated charges of signals for ID-PMTs and the OD-PMTs. The QBEE provides high-speed signal processing by combining pipelined components, these included a newly developed custom charge-to-time converter (QTC) 
in the form of an application-specific integrated circuit (ASIC), a multi-hit time-to-digital converter (TDC), and field-programmable gate array (FPGA) [9]. Each QTC input has three gain ranges - "Small", "Medium" and "Large" - the resolutions for each are shown in Table 2 . For each range, analog to digital conversion is conducted separately, but the only range used is that with the highest resolution that is not being saturated. The overall charge dynamic range of the QTC is $0.2 \sim 2500 \mathrm{pC}$, which is about five times larger than that of the old ATM. The charge and timing resolution of the QBEE at the single photoelectron level is 0.1 photoelectrons and $0.3 \mathrm{~ns}$ respectively, both are better than the intrinsic resolution of the 20-inch PMTs used in SK. The QBEE achieves good charge linearity over a wide dynamic range. The integrated charge linearity of the electronics is better than $1 \%$. The thresholds of the discriminators in the QTC are set to $-0.69 \mathrm{mV}$ (equivalent to 0.25 photoelectron, which is the same as for SK-III). This threshold was chosen to replicate the behavior of the detector during its previous ATM-based phases.

\begin{tabular}{|c|c|c|}
\hline Range & Measuring Region & Resolution \\
\hline Small & $0-51 \mathrm{pC}$ & $0.1 \mathrm{pC} /$ count $(0.04 \mathrm{pe} /$ count $)$ \\
\hline Medium & $0-357 \mathrm{pC}$ & $0.7 \mathrm{pC} /$ count $(0.26 \mathrm{pe} /$ count $)$ \\
\hline Large & $0-2500 \mathrm{pC}$ & $4.9 \mathrm{pC} /$ count $(1.8 \mathrm{pe} /$ count $)$ \\
\hline
\end{tabular}

Table 2: Summary of QTC ranges for charge acquisition.

\subsection{Water tank}

\subsubsection{Tank geometry}

During the reconstruction period between SK-II and SK-III, we surveyed the tank to verify the blueprint-based MC implementation of the geometry. The detector surface is subdivided into a barrel part and two round parts, top and bottom part. The positions of 14 PMTs (ten around the center of the barrel at various azimuthal positions, three PMTs at top and one at bottom) were measured using a transit theolodite. All were consistent with their respective design values up to a few $\mathrm{cm}$. This difference was small enough that we did not need to alter fiducial volume estimates.

\subsubsection{Geomagnetic field inside the tank}

As described in [1], 26 sets of horizontal and vertical coils are arranged around the inner surface of the tank to neutralize the geomagnetic field that would otherwise affect photoelectron trajectories in the PMTs. A $100 \mathrm{mG}$ field applied parallel to the dynode plate of a 20-inch PMT reduces the hit collection efficiency by $10 \%$, and it does so in an asymmetric manner [10].

Before and during filling of the tank with water for SK-III, and with the coils carrying their design currents, the residual fields at 458 PMT locations around the detector were measured using a device ${ }^{13}$ that can simultaneously measure the magnetic field vector along three orthogonal axes. The left side of Fig. 1 shows the distribution of measured field intensities. The average field intensity is $32 \mathrm{mG}$, which corresponds, on average, to $20 \mathrm{mG}$ along the PMT dynode axis, and results in $2 \%$ deviation in the collection efficiency of a photoelectron. The right side of Fig. 11 shows the magnetic field along each detector axis. The average vertical (z) component is nearly

\footnotetext{
${ }^{13}$ FGM-4DTAM, Walker LDJ Scientific, inc.
} 
zero, but the horizontal components ( $\mathrm{x}$ and $\mathrm{y}$ ) show a $10 \mathrm{mG}$ shift resulting from overcompensation of the geomagnetic field 14 . Figure 2 shows field measurements at the top, center and bottom of the detector. The top and bottom exhibit similar field patterns, with somewhat higher residual fields than in the barrel. From these studies we estimate that the effect of those residual fields on the collection efficiency of the ID-PMTs is about $1 \sim 2 \%$.
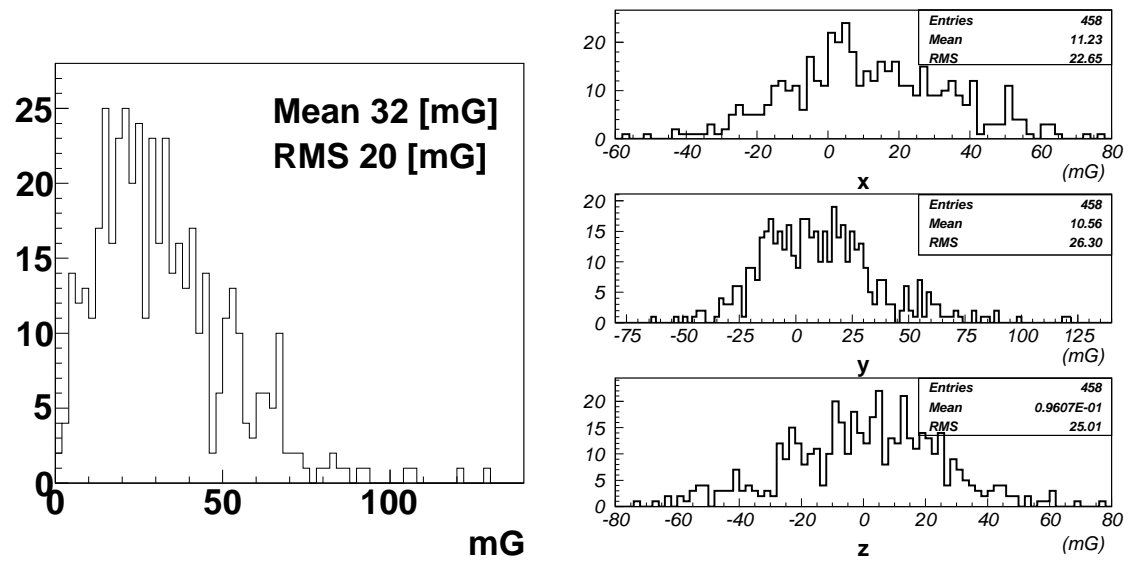

Figure 1: Distribution of magnitude of the residual magnetic field at different locations in the detector. The left figure shows the magnitude; the right figures show the value along the usual SK coordinate system axes.
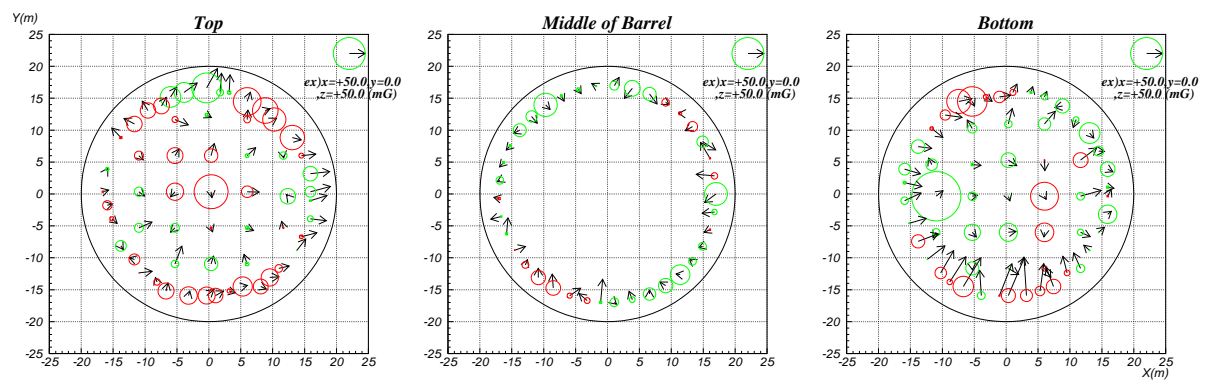

Figure 2: Intensities (represented by size of the circle) and directions (represented by an arrow for horizontal; blue solid shows upward and red dashed shows downward) of the remaining magnetic field. The three panels show the results of field measurements at PMT positions at the top, around the center, and at the bottom of the ID. The blue circle and the arrow in the top right show the reference scale for a $50 \mathrm{mG}$ field in both the $\mathrm{x}$ and $\mathrm{z}$ direction, respectively.

\subsection{Water circulation}

Understanding the condition of the water in the SK detector is crucial for proper calibration of the detector because the water condition affects photon propagation in the tank. The water used

\footnotetext{
${ }^{14}$ The direction of magnetic north corresponds to $(-0.74,-0.68)$.
} 
in SK is sourced from natural water inside the Kamioka mine. It is purified by a dedicated system and it is continually recirculated through parts of that system during normal detector operation. Figure 3 shows a schematic of the circulation system. To monitor water temperatures and their time dependence, thermometers with $0.0001^{\circ}$ precision ${ }^{15}$ are placed at eight positions in both the ID and the OD.

In the ID, the water circulation pattern can be manipulated by changing the temperature of the water that is fed into the detector from the re-circulation system.

In the usual case, we find that water is always convecting below $\mathrm{z}=-11 \mathrm{~m}$, which results in uniform temperature throughout that lower part of the ID. Above that level, water is layered, and its temperature gradually rises, resulting in a $0.2^{\circ}$ difference between the top of the ID and the convection zone in its lower part (see Fig. 4). The water in this layered region slowly rises through these layers over the course of one month. This results in a $5 \%$ difference in water transparency over the detector, as discussed in Section 3.2.1

While we can expand the convective region that normally resides at the bottom of the ID to cover the full ID volume, then the water quality would be uniform. When convection extends over the whole ID volume, the water temperature difference becomes less than $0.01^{\circ}$. This small temperature difference clearly indicates full convection of water in the SK tank and thus indicates the best uniformity in optical properties of water in the ID. This water condition period was used for measurement of relative differences of quantum efficiency in each PMTs as described in Section 3.1.5.

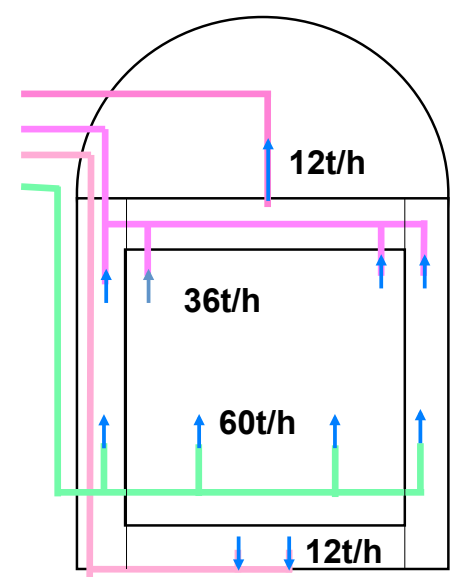

Figure 3: Diagram of the water circulation system in SK-IV.

\footnotetext{
${ }^{15}$ Denko Co., QT-860S, Yokohama, Japan, Tel:+81-453317089, Fax:+81-453317284.
} 


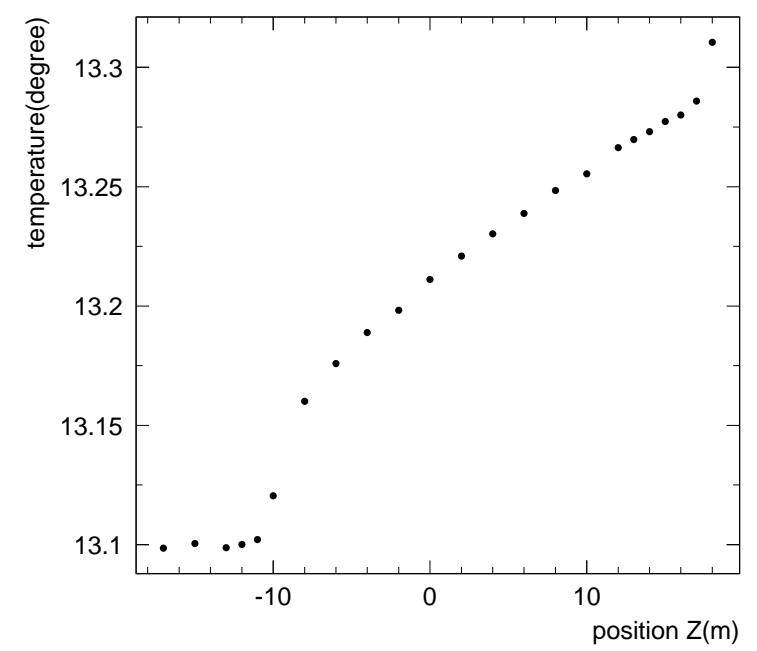

Figure 4: The vertical dependence of the water temperature in the ID.

\section{Inner detector calibration}

\subsection{PMT and electronics calibrations}

\subsubsection{Introduction}

To provide background for this section, a brief description of PMT calibration is presented here. The 20-inch diameter PMTs developed by Hamamatsu Photonics K.K. (R3600-05(A)) [10] are used in the inner detector. These PMTs have a photo-cathode made of bialkali (Sb-K-Cs), and has its maximal photon conversion probability in the wavelength range of Cherenkov light. The PMT dynodes are of a Venetian-blind type, and their base circuit is an optimized 11-stage voltage divider. The high-voltage system for the PMTs was manufactured by CAEN Co. and consists of distributors (A933K), controllers (SY527), and interface modules (V288).

Since the timing behavior of PMTs depends on the charge of the measured pulse, we begin discussing ID-PMT calibrations with charge-related issues. In the definition for the PMT charge calibration, "gain" is a conversion factor from the number of photoelectrons to charge (in units of $\mathrm{pC}$ ), and "QE" is the product of the quantum efficiency and collection efficiency of photoelectrons onto the first dynode of the PMT. Low-energy physics events like solar neutrinos largely consist of single-photoelectron (single-pe) hits and rely heavily on the QE calibration for their interpretation, whereas high-energy events like those involving $\mathrm{TeV}$-scale muons depend more on proper gain calibration. Knowledge of both gain and QE is important and must be available on a PMT-by-PMT basis.

Unfortunately, the old ATMs used in SK-I, II, and III did not allow us to record meaningful single-pe distributions on a PMT-by-PMT basis, however, a cumulative distribution for all PMTs could be obtained after the relative gains had been properly calibrated.

This situation forces us to set up PMT calibration in the following way. First, we need to determine a suitable high-voltage value to be applied to each ID-PMT. This determination is described in Section 3.1.2. Next we need to understand the differences in gain between individual 
ID-PMTs. Section 3.1.3 details this effort and its results. Once we are able to obtain meaningful cumulative single-pe distribution for all ID-PMTs, Section 3.1.4 describes how to use this cumulative single-pe distribution to calibrate the average gain over all ID-PMTs. Referencing, in turn, the gain variation for an individual PMT to the average gain gives the individual gain of each ID-PMT. In Section 3.1.5 we use Monte Carlo simulations to extract a calibration of the QE for each individual PMT. This new procedure which determines the gain and QE of an ID-PMT's independently is a major improvement over the procedure used previously. Section 3.1.6 describes the validation of both the gain and QE calibrations, including verifications of their consistency. Discussion of charge-related calibration issues is concluded in Section 3.1.7, which describes measurements for assessing the linearity of charge determinations. Section 3.1.8 addresses the ID-PMT timing calibration.

These calibrations, except for the establishment of 420 reference PMTs, were performed in the beginning of SK-I, II and III. In addition, a real-time calibration system monitors crucial parameters throughout normal operations of the experiment to allow us to consider variability as well as ensure stability during data-taking. For this purpose, light sources are permanently deployed near the center of the ID. During SK data-taking, the lights flash in turn at approximately 1-s intervals. As detailed in subsections 3.1.2, 3.1.8, and 3.2.1, they monitor ID-PMT gains and timing as well as optical parameters of ID water.

\subsubsection{Determination of the high-voltage setting for each PMT}

To establish the hith-voltage (HV) setting for each PMT, we require that all PMTs give the same output charge for the same incident light intensity. For this purpose, an isotropic light source is placed at the center of the SK tank. Since the SK tank is a large cylinder about $40 \mathrm{~m}$ in both diameter and height, we expect the amount of light reaching each PMT from that source to be about a factor of two different between the closest and farthest PMT. Correcting for only this geometrical difference is insufficient, because it ignores important corrections, such as photon propagation in the water, which depends on water quality and reflections from ID surfaces. To avoid such problems, we prepared 420 "standard PMTs" for which HV values were determined individually before installation during SK-II and SK-III, these determinations used the pre-calibration system shown in Fig. 5. The standard PMTs were later mounted in the ID and served as references for other PMTs that were grouped based on similar geometrical relationships to the light source at the detector center. (See also Fig. 8)

In the pre-calibration system, the light from a Xe lamp was passed through a UV filter and then injected simultaneously into three optical fibers. One fiber went to a "scintillator ball" placed in a dark light-tight box. The other two fibers went to avalanche photodiode (APD) modules, which monitored the light intensity of the Xe lamp. The scintillator ball ${ }^{16}$ is a $5 \mathrm{~cm}$ diameter acrylic ball containing $15 \mathrm{ppm}$ of POPOP as a wavelength shifter and $2000 \mathrm{ppm}$ of $\mathrm{MgO}$ as a diffuser to make the light emission from the ball as uniform as possible. Its uniformity in phi was measured to be better than $1 \%$. The dark box was made of $\mu$-metal to shield against the geomagnetic field. The residual field inside the dark box was measured to be less than $20 \mathrm{mG}$. Two 2-inch PMTs were also mounted inside that box to monitor the light intensity from the scintillator ball. The HV values of the 420 PMTs were adjusted so that the observed ADC counts returned the target $\mathrm{ADC}$ value for the reference about 30 photoelectrons for the light intensity from the scintillator ball.

\footnotetext{
${ }^{16}$ Manufactured by CI Kogyo, Saitama, Japan, Tel:+81-429481811, Fax:+81-429491395.
} 
These HV adjustments were completed over a two-week period inside the Kamioka mine. To monitor the stability of the adjustment procedure, the same PMT was tested every morning and every evening, and variations were found to be smaller than $1 \%$. The temperature inside the dark box was also monitored, and its variation was less than 0.1 degre ${ }^{17}$ The measurements were done by first applying four different HV values. Figure 6 shows a typical gain curve. Applying a linear fit to this plot, the HV value that gave the target ADC counts was determined. Finally, the obtained HV was applied, and it was confirmed that the observed ADC counts were within $1 \%$ of the target value. Reproducibility was also checked. At the end of the measurement, we selected 50 PMTs randomly from the 420 PMTs and repeated the above procedure on them. Figure 7 shows fractional differences between the results of the first and second $\mathrm{HV}$ adjustments. The reproducibility was within $1.3 \%$ RMS.

The 420 standard PMTs were placed as shown by the red points in Fig. 8 to serve as references for other PMTs that would have similar geometrical relationships to the light source at the center of the ID. Examples of such groupings are shown on the right in Fig. 8. The HV settings of those other PMTs were adjusted so that the observed charges from the light flashes of the light source ${ }^{18}$ were matched to the average charges for those standard PMTs having a similar geometrical relationship to the light source, as shown in Fig. 8. This HV adjustment was done at the beginning of SK-III, just after installing all PMTs into the detector. The same scintillator ball/Xe lamp combination was used in this step as had been used in the pre-calibration. After determining proper HV settings for all PMTs, we checked reproducibility. Figure 9 shows the percent differences in observed charge with respect to the reference value. Good reproducibility, within $1.3 \%$ RMS, was found, this was consistent with the result from the pre-calibration. The scintillator ball/Xe lamp light source remains permanently at the center of the ID for real-time as well as long-term monitoring of ID-PMT gains.

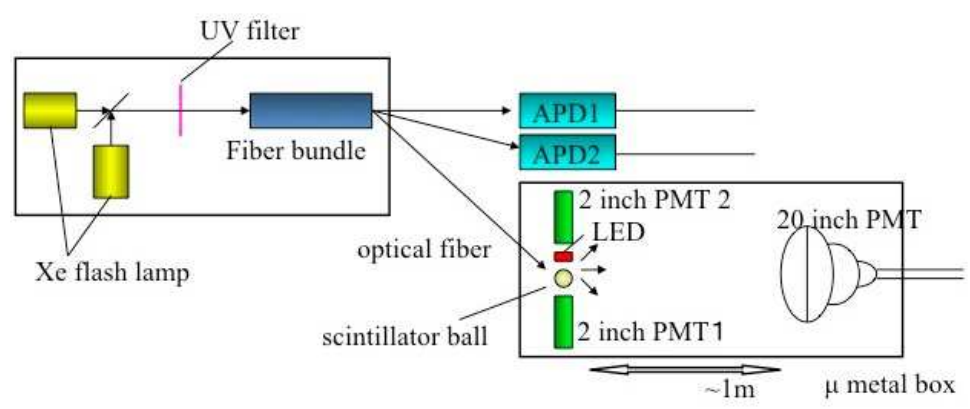

Figure 5: Schematic view of the setup for the pre-calibration. A Xe flash lamp, placed inside a box, emitted light that was guided by optical fibers through a fiber bundle to two avalanche photodiodes and a scintillator ball located in another $\mu$-metal shielded dark box, where a 20 inch PMT was exposed to the light from the scintillator ball. Two 2-inch PMTs monitor the light output of the scintillator ball.

\footnotetext{
${ }^{17}$ This could be achieved since all work was done inside the Kamioka mine where ambient temperature is quite stable.

${ }^{18}$ The accuracy of the position of the light source was within $\pm 1 \mathrm{~cm}$ at the detector center.
} 


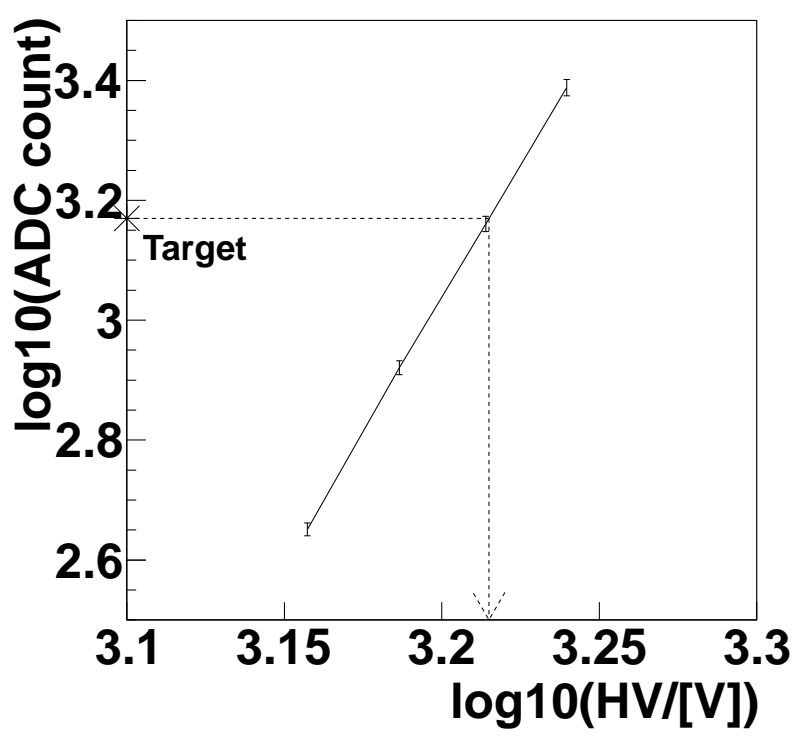

Figure 6: Typical gain curve. The horizontal axis is HV value and the vertical axis is ADC counts. Both axes are in log scale. After measurements with four different HV values, the HV for the target ADC count was calculated by fitting the four points by an analytical formula $y=\alpha x^{\beta}$, where $\alpha$ and $\beta$ are constants for each PMT. An additional measurement with that $\mathrm{HV}$ value was performed for confirmation.

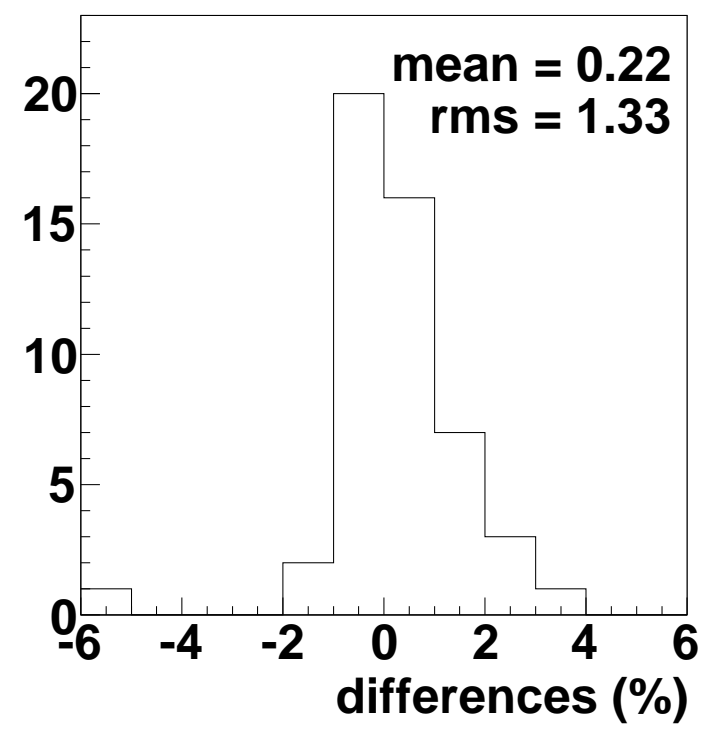

Figure 7: Distribution of the observed charge differences between the first and second measurements in pre-calibration for checking reproducibility. 50 out of 420 standard PMTs had been selected at random for this check. 

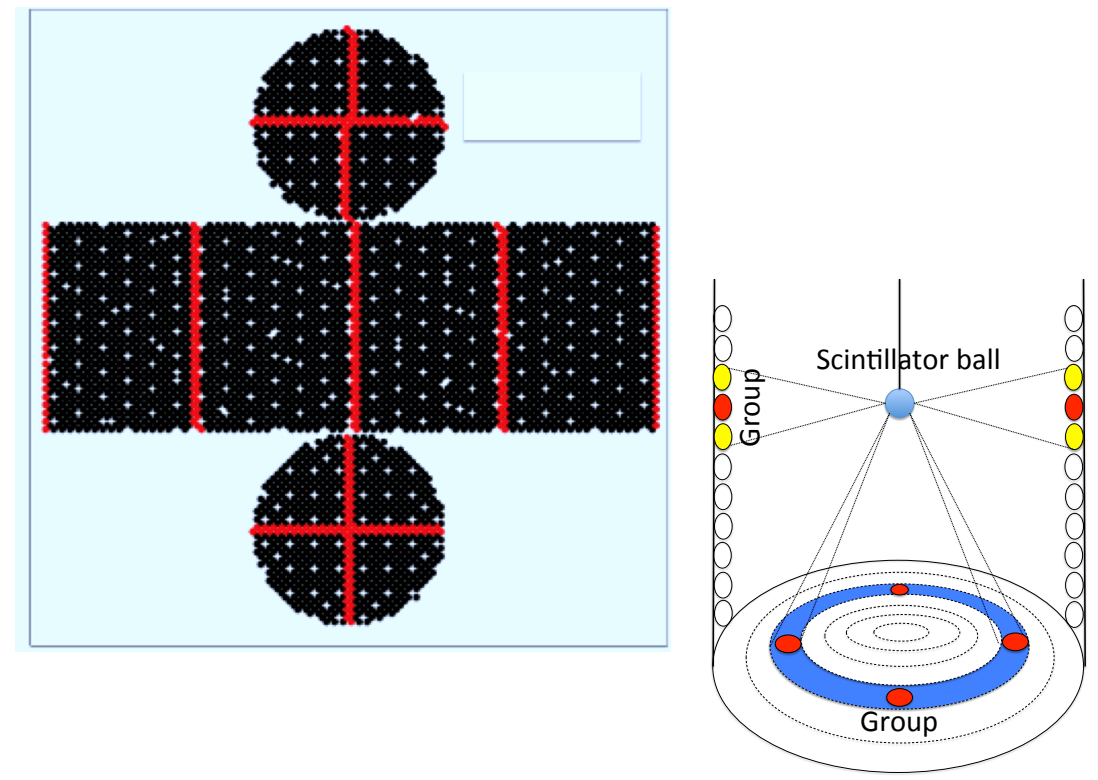

Figure 8: The location of "standard PMTs" inside the SK inner detector (left). The red points indicate the locations of the standard PMTs. These PMTs served as references for other PMTs belonging to the same group with similar geometrical relationship to the light source (right). 


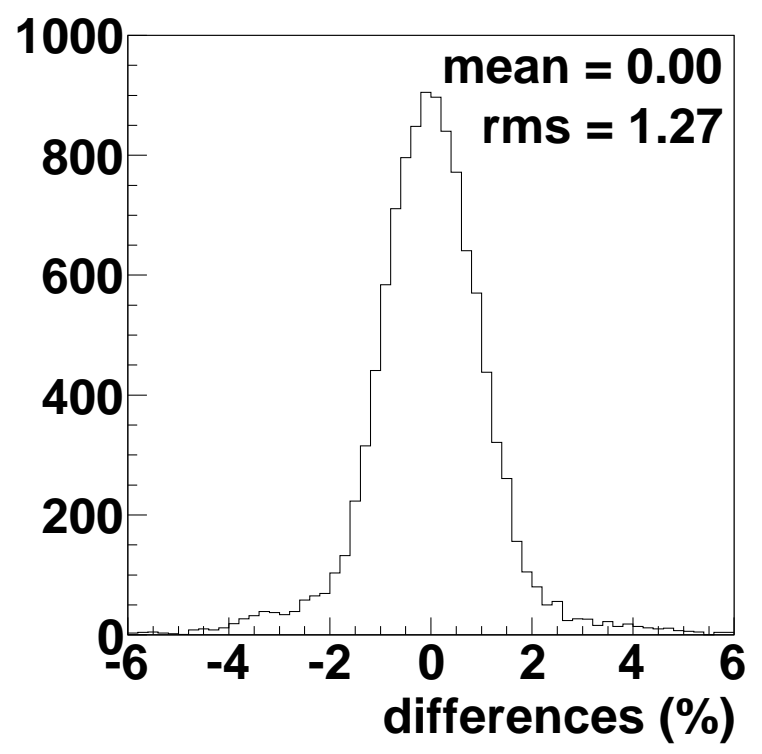

Figure 9: The observed percent charge differences for all ID-PMTs from their respective reference value. 


\subsubsection{Relative differences in gain}

To interpret the output charge from the PMTs in number of photoelectrons, we need to determine the gain for each PMT and is done in two steps. The first step is to derive the relative difference among PMTs (described in this section). Based on this first step we can then determine the average gain over the whole detector (described in the next section). Once we know the average and the deviation from that average for each individual ID-PMT, we can extract the gain of each individual ID-PMT.

To measure of the relative gain difference, a stable light source emitting constant-intensity flashes is deployed at a certain position in the tank. There are two measurements: the first uses high-intensity flashes from which every PMT gets a suitable number of photons, creating an average charge $Q_{o b s}(i)$ for each ID-PMT $i$. The second measurement uses low-intensity flashes in which only a few PMTs are hit in each event, therefore, we can be reasonably sure that each of these is a single-pe hit. We count the number of times $N_{o b s}(i)$ that PMT $i$ records a charge that is greater than the threshold value. Since the location of the light source is not changed between the two measurements, the complicating factors in estimating those two intensities $Q_{o b s}(i)$ and $N_{o b s}(i)$ are almost identical:

$$
\begin{aligned}
& Q_{o b s}(i) \quad \propto \quad I_{s} \times a(i) \times \varepsilon_{q e}(i) \times G(i), \\
& N_{\text {obs }}(i) \quad \propto \quad I_{w} \times a(i) \times \varepsilon_{q e}(i),
\end{aligned}
$$

where $I_{s}$ and $I_{w}$ are the average intensities of high and low intensity flashes, respectively, $a(i)$ is the acceptance of ID-PMT $i, \varepsilon_{q e}$ denotes its QE, and $G(i)$ its gain. The threshold is sufficiently low that the relative changes in gain, which we want to track, have little effect on $N_{o b s}(i)$, for example, $10 \%$ gain change makes the $N_{\text {obs }}(i)$ just $1.5 \%$ change. The low threshold enables us to ignore, in the above calculations, differences in probability for having a charge below the discriminator threshold among PMTs. The gain of each PMT can then be derived by taking the ratio of Eq. (1) and (2), except for a factor common to all PMTs:

$$
G(i) \propto \frac{Q_{o b s}(i)}{N_{o b s}(i)} .
$$

Then the relative gain of each ID-PMT can be obtained by normalization with the average gain over all PMTs 19

To perform this calibration we need a means to change the intensity of the flashes of the light source. The light source is nitrogen-laser-driven dye laser (Section 3.1.8). To manipulate the overall intensity of the light delivered into the ID, we used a filter wheel with neutral density filters between the dye laser, and the optical fiber that feeds light into the diffuser ball.

Figure 10 shows the ratio (3) for each PMT, the RMS of the distribution was found to be $5.9 \%$. Since the HV value for each PMT was determined to make $Q_{o b s}$ be the same, we infer that this deviation is due to differences in QE among PMTs. The observed ratio in Eq. (3) for each PMT, normalized by the average over all PMTs, contributed to a table of relative gain differences among PMTs. These factors for relative gain differences of each PMT are used as fine corrections in conversion from output charge to number of photoelectrons observed.

\footnotetext{
${ }^{19}$ The common factor $I_{s} / I_{w}$ is also eliminated by this normalization. In the actual measurement, $N_{o b s}$ was corrected by occupancy.
} 


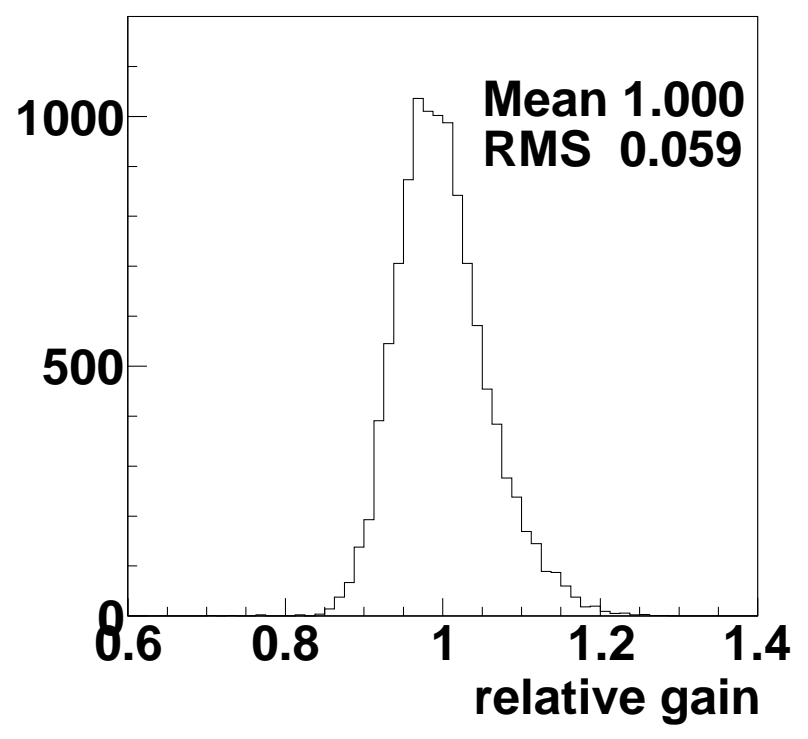

Figure 10: Distribution of relative gain of PMTs.

\subsubsection{Absolute gain conversion factor}

As pointed out previously, the relative gain for a PMT is usually obtained from the average of its single-pe distribution. Problems with pedestal subtraction in the ATMs before SK-IV prevented us from doing this on a PMT-by-PMT basis. Given the continuous distribution of relative gain corrections obtained in the previous section, we can build the cumulative single-pe distribution for all ID-PMTs. Applying the relative gain correction aligns the single-pe spectra of all ID-PMTs so that it makes sense to add them, it also effectively smoothes the sampling of this distribution enough to overcome problems encountered at the single-PMT level. While some additional smearing is introduced by the intrinsic resolution of the relative gain calibration, the resulting cumulative single-pe distribution largely represents the average single-pe response of the detector. In particular, we can extract from it the absolute gain of all ID-PMTs, which had been normalized out when we calibrated the relative gains. In this section, we describe the data we used and present the results of absolute gain calibration.

For this measurement, a uniform and stable source of single-photoelectron level light is required. We used a "nickel source" that isotropically emits gamma rays. The gamma rays have about $9 \mathrm{MeV}$ from thermal neutron capture on nickel from the reaction ${ }^{58} \mathrm{Ni}(n, \gamma)^{59} \mathrm{Ni}$. A ${ }^{252} \mathrm{Cf}$ source provides neutrons. More details can be found in Section 8.7 of [1]. The cylindrical geometry and inhomogeneous nickel distribution of the old nickel source led us to build a new one with significantly improved symmetry. (Figure 11) Deployed at the center of the ID, this source delivers 0.004 pe/PMT/event on average, a level at which more than $99 \%$ of observed signals are due to single-pe.

The nickel source measurement was done at the beginning of SK-III. The resulting charge distribution is shown in the histogram in Fig. 12 that was obtained after correcting for relative gain differences, as described in Section 3.1.3 and accumulating data from all ID-PMTs. To 


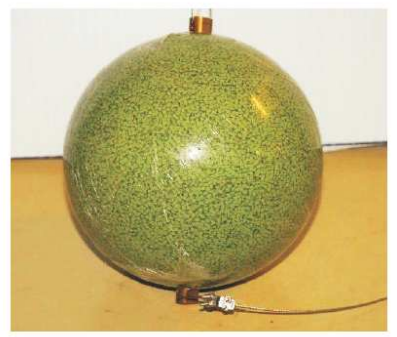

Figure 11: Picture of the nickel source which was manufactured by CI Kogyo. The ball was made of $6.5 \mathrm{~kg}$ of $\mathrm{NiO}$ and $3.5 \mathrm{~kg}$ of polyethylene. The $\mathrm{Cf}$ source was inserted into the center of the ball and held there by a brass rod.

minimize the effect of dark hits, a similar distribution was made using off-time (the timing window in which we do not expect a signal) data and subtracting it from on-time (timing window in which we do expect a signal) data. To evaluate the distribution below the usual threshold of 0.25 photoelectron, data with higher PMT gain and lower discrimination threshold were obtained. The dashed histogram in Fig. 12 shows the data with double the usual PMT gain and half the usual discrimination threshold. Since it was not possible to obtain data in the region less than $0.3 \mathrm{pC}$, we used a straight-line extrapolation into this low-charge region. The systematic uncertainty introduced by this assumption below $0.3 \mathrm{pC}$ becomes negligible after considering the true discrimination threshold and the small amount of charge. The value averaged over the whole $\mathrm{pC}$ region was defined as the conversion factor from $\mathrm{pC}$ to single-pe; the value of this conversion factor was $2.243 \mathrm{pC}$ to single-pe. At the beginning of SK-IV, we repeated this measurement and found the new conversion factor to be $2.658 \mathrm{pC}$ per photoelectron. This difference comes from a long-term increase in the PMT gain. No clear reason has been identified for this increase, but it is accounted for in physics analyses.

The single-pe distribution, as constructed above, is also implemented in MC simulations. The solid line in Fig. 13 is the same as the one we pieced together in Fig. 12, with the axis converted from $\mathrm{pC}$ to photoelectron. For simulations of multiple photons in ID-PMTs, we sum values drawn from this distribution. The nickel-source data are also used to extract the threshold behavior for MC simulations. The dashed histogram in Fig. 13 is the experimentally-observed distribution and has the threshold folded into it. In MC simulations, we use the ratio of the observed (dashed) and partly observed, partly extrapolated (solid) histograms in Fig. 13 to implement single-hit threshold behavior.

\subsubsection{Relative differences in QES}

Values for QE differ from PMT to PMT. Here we describe how we determine the relative QE for each PMT. If the intensity of a light source is low enough, the observed hit probability should be proportional to the value of QE for the PMT, as can be seen from Eq. (2). While we can count the number of hits measured by each PMT, we cannot easily determine how many photons reached it. Therefore, we used MC simulation to predict the number of photons arriving at each PMT, and took the ratio of the observed number of hits to predicted number of hits.

For this measurement, we use the nickel source used in absolute gain measurements (Section 3.1.4). In addition, the uniformity of water quality throughout the tank is quite important, since any non uniformity in water properties causes the hit probability to depend on the PMT 

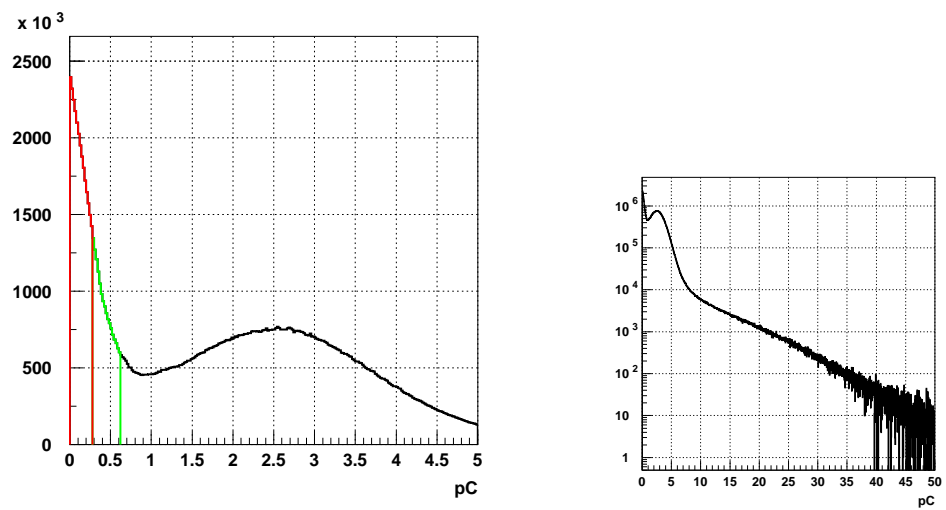

Figure 12: The single-pe distributions in $\mathrm{pC}$ unit for nickel source data in SK-III. The right plot shows the same histogram in logarithmic scale. The solid line in the left figure shows the data with normal PMT gain, the dashed line shows the data with double gain and half threshold, and the dotted line is linear extrapolation.

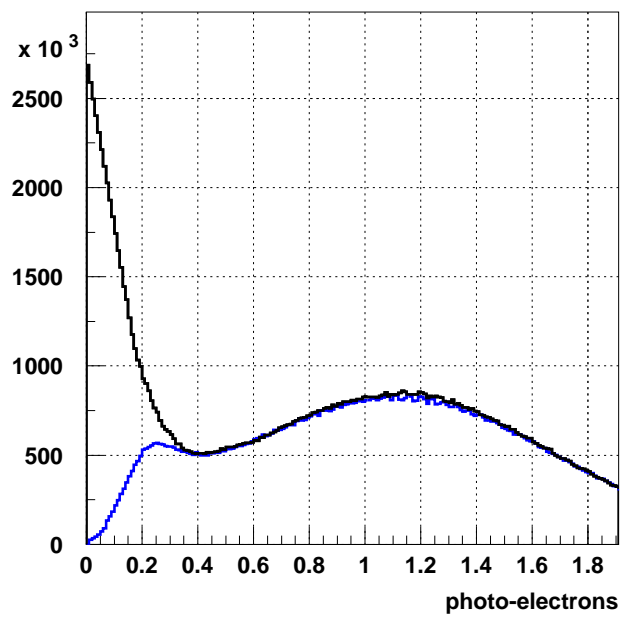

Figure 13: The single-pe distributions for our MC simulation (solid line). This distribution was implemented while we were still using the ATM-based electronics. The dashed line shows the distribution of number of photoelectrons from the nickel data in SK-IV. The difference between them is due to the threshold function of the QBEE, and the ratio is also put into our MC simulation.

position not just because of relative geometry, but also because of the exact conditions along the photon path. As discussed in Section 2.4, this condition can be identified by measuring the 
temperature profile throughout the ID. We conducted this calibration when the water convected over the whole ID volume 20 It was also confirmed that no significant top-bottom asymmetry of water quality existed because no differences in the standard PMTs appeared between top and bottom. We used nickel source data from that day for this measurement.

Figure 14 shows the position dependence of the hit probability with the following corrections:

$$
N_{o b s}(i) \times R(i)^{2} / a(\theta(i))
$$

where $i$ again indexes the ID-PMTs, $R(i)$ is the distance from the source position to the PMT position, and $a(\theta)$ is the acceptance as a function of incident angle $\theta$ [4]. Even after this correction, some position dependence still remains because of reflection from neighboring surfaces, plus scattering and absorption by the water. These further corrections were estimated through Monte Carlo simulations, which considered absorption and scattering in water and reflection from the surfaces of the PMTs and the black sheet. Once these light propagation effects, as estimated by the MC simulations, are considered, the remaining difference is attributed to the QE of individual PMTs. To remove the dependence of the absolute light intensity from the calibration, we normalize taking the average of these residuals to obtain a measure for the relative $\mathrm{QE}$ of the ID-PMTs. This quantity is tabulated for use in the MC simulations.

The cause of these relative differences among PMTs is interesting. One cause is, of course, an irreducible variability in the manufacturing process for the PMTs. We also found some systematic changes related to the time of manufacturing of the PMTs. To see this, the PMTs were categorized into two groups: those used in SK-II, and the newly produced PMTs for the reconstruction of SK after the accident. The latter were first installed in SK-III. Figure 15 shows a plot similar to Fig. 14, but divided into the two categories; a clear difference can be seen. First, among the PMTs used in SK-II, the bottom PMTs have 4.5\% higher QEs than the top ones. These PMTs came from different production runs: the PMTs installed in the bottom region were produced in 1996 and 1997, while those in the top and barrel were produced between 1992 and 1995. The newer PMTs have higher QEs. An even clearer difference can be seen if we compare PMTs for SK-II and SK-III. The origin of this systematic difference was traced to the fact that the manufacturer, Hamamatsu Photonics K.K., continued to improve the transparency of the glass envelope. All the tendencies we observed in the QE measurements are supported by independent measurements of glass properties at the manufacturer.

These findings also have implications for the interpretation of the width of the relative gain distribution in Fig. 10. As described in Section 3.1.2, we balanced the response of the ID-PMTs by adjusting their HV so that, at some light level, each PMT $i$ responded with the same charge $Q_{o b s}(i)$, which can be calculated from its gain and QE in Eq. (1). According to that equation, the charge produced by the PMT is proportional to the product of QE and gain. Because we know that there are systematic differences in QEs between the PMTs in the detector, we also know that the way we adjusted the HV for an individual tube compensated for a lower (higher) QE by requiring a higher (lower) gain (higher (lower) HV) to deliver the same charge at the same light level Later, as described in Section 3.1.3, we took the ratio of a similar set of high-light-level data to low-light-level data for each tube to eliminate acceptance and QE effects, thereby obtaining the PMT relative gain, the distribution of these relative gains is shown in Fig. 10 In the next section we show that the procedures discussed above produce a well-balanced detector response.

\footnotetext{
${ }^{20}$ the data were taken on October 12, 2006 when was just after SK-III started.
} 

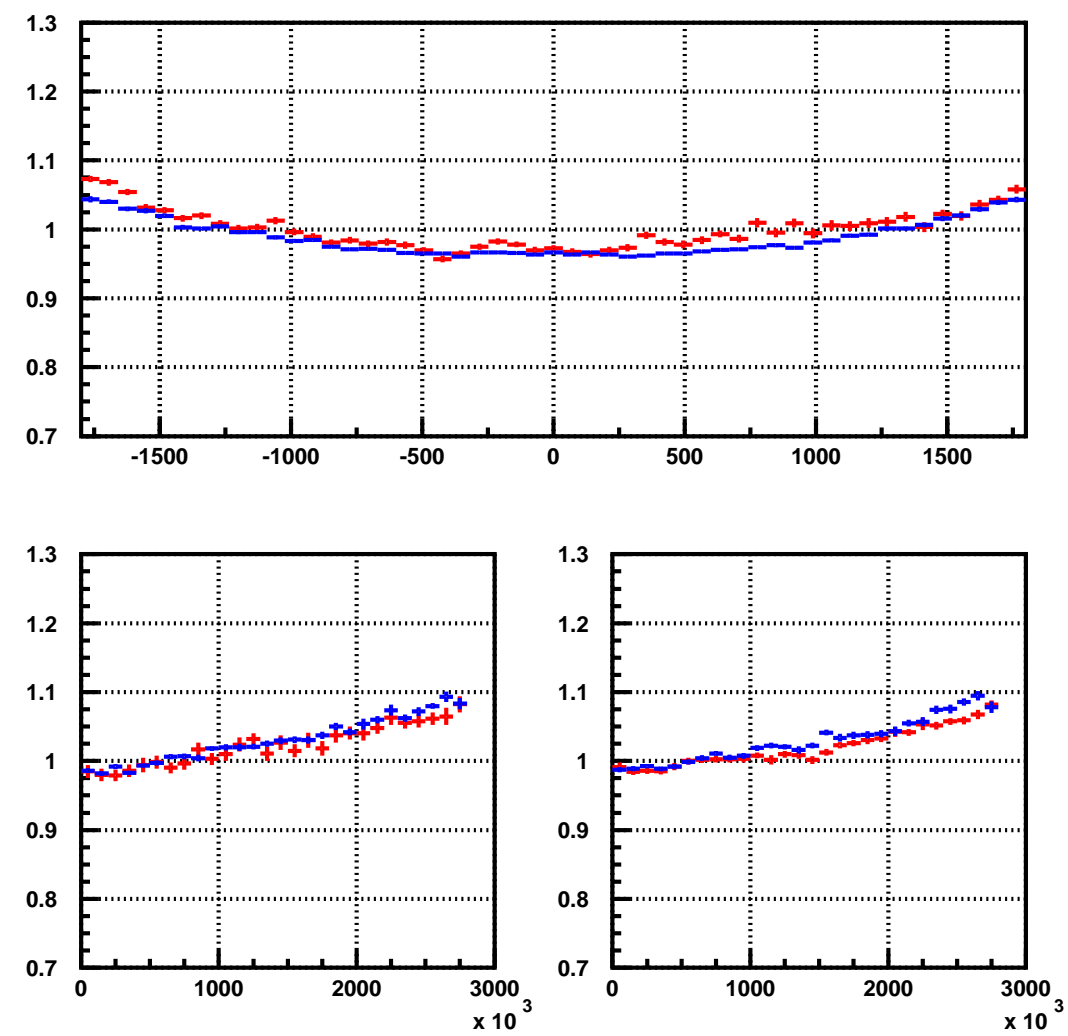

Figure 14: Hit probability as a function of PMT position. The vertical axis shows the number of hits normalized by average value of all the PMTs. The upper figure shows the barrel PMTs where the horizontal axis denotes the $\mathrm{z}(\mathrm{cm})$ position of PMTs. The lower figures show top (left) and bottom (right) PMTs, where the horizontal axis shows the square of the distance from the center $\left(\mathrm{cm}^{2}\right)$. The red thick line shows the data, and the blue thin line shows MC. The MC was not corrected by QE differences in each PMTs. 

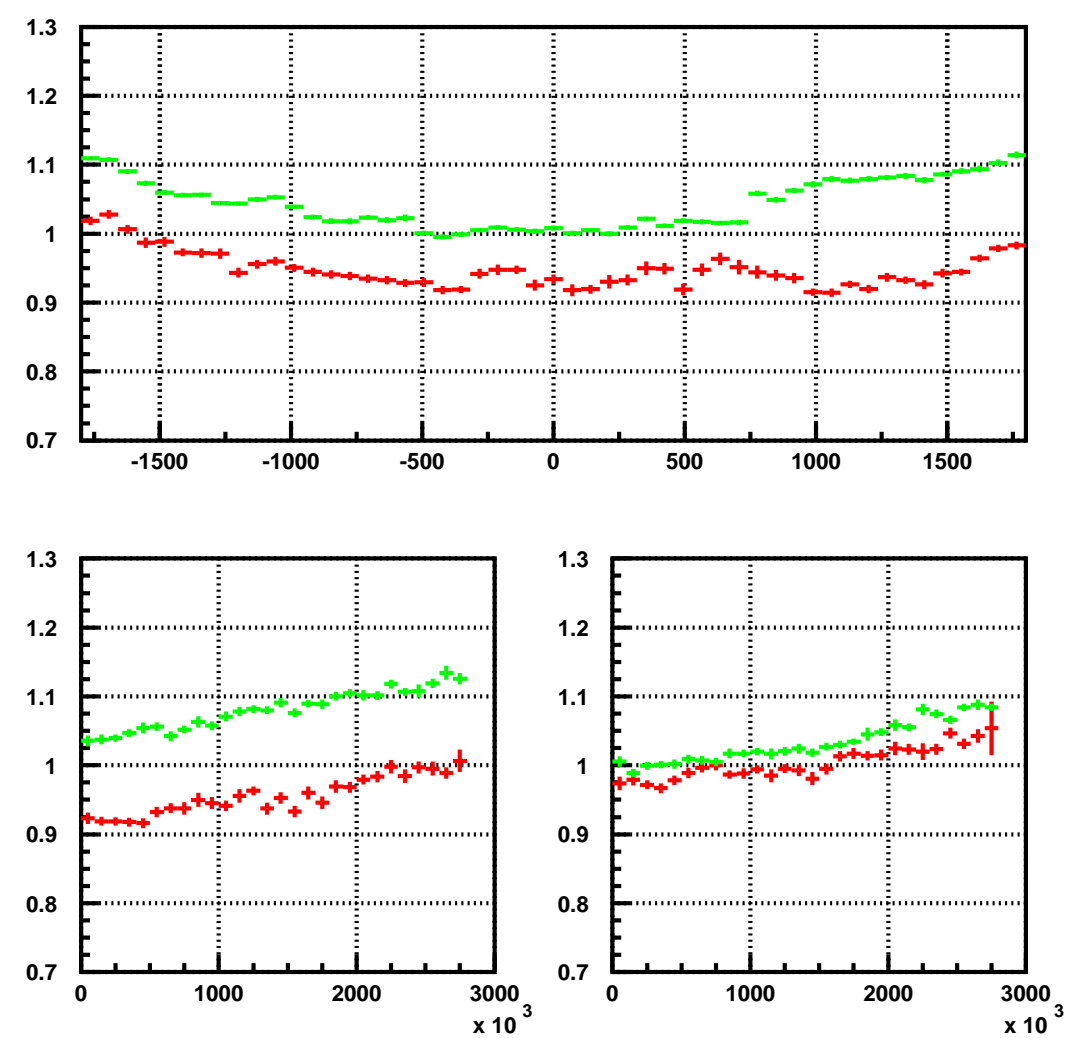

Figure 15: Normalized hit probability as a function of PMT position, similar to Fig. 14 The red thick line shows the PMTs used in SK-II, and the green thin line shows the PMTs newly installed from SK-III. 


\subsubsection{Overall symmetry of detector response}

From the discussion in the previous section, we expect an anti-correlation between the relative gains and the QEs that our procedures established for the ID-PMTs and this anti-correlation Fig. 16 From the previous discussion, we also know that the distribution of PMTs with intrinsically higher $\mathrm{QE}$ is not uniform over the detector, since the water pressure at the bottom of the tank is significantly higher than at the top, we systematically installed the new and presumably less-stressed PMTs at the bottom and concentrated the older PMTs at the top of the detector. In this section we describe a dedicated experiment that was designed to assess whether independent calibrations of the two anti-correlated quantities combined with this non uniform distribution inside the detector result in any residual non uniformity.

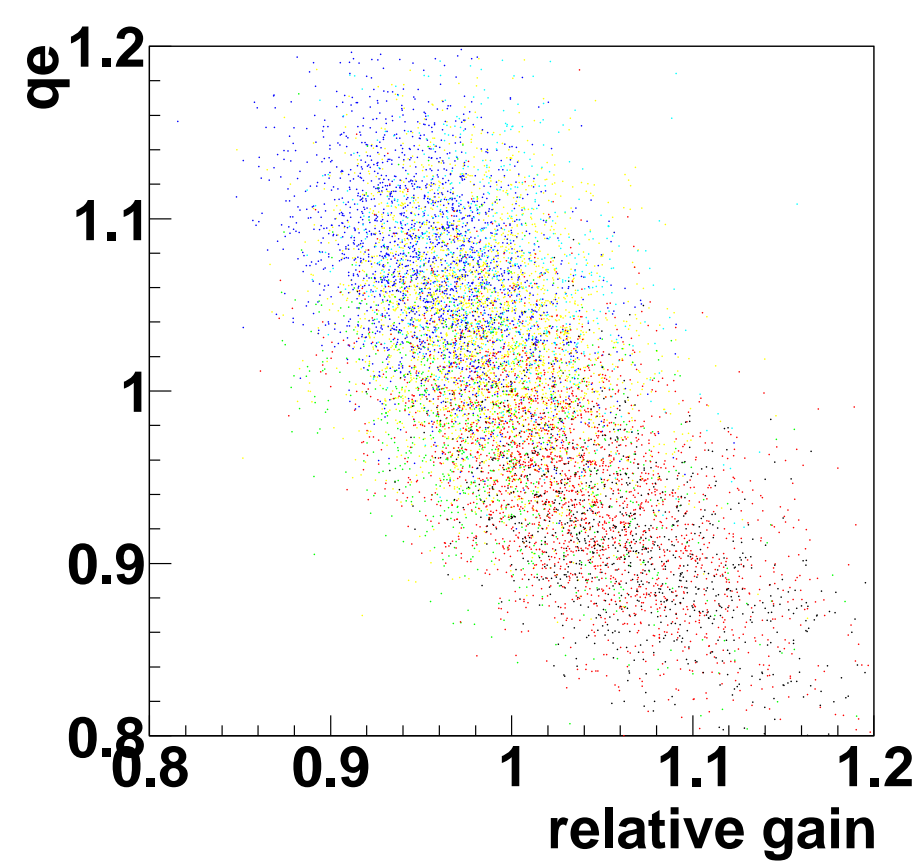

Figure 16: The relationship between the relative gains and the QEs. The color shows the PMT production period: black 1992-1993, red 1994-1995, green 1996-1997, sky-blue 2003, blue 2005, and yellow 2006.

The left side of Fig. 17 shows the setup for this experiment. The light source was the same one used to determine the HV settings: the scintillator ball driven by Xe flash lamp. As pointed out in Section 3.1.2, the light from the Xe flash lamp is injected into the scintillator via an optical fiber. In this measurement, we are mostly concerned with a possible top-bottom asymmetry in the detector response, therefore, we mounted the scintillator ball so that the fiber entered the ball in the horizontal rather than the usual vertical direction (the fiber normally enters straight from above). In this way, any residual forward/backward asymmetry in the light output from the scintillator ball would affect the wall region of the ID rather than the top and bottom. In contrast, during the original $\mathrm{HV}$-setting procedure such an asymmetry would affect the top and bottom regions.

The right side of Fig. 17 shows the results from this measurement. This data was obtained 
the day before the nickel-source measurement ${ }^{21}$ The data confirmed that convection occurred and no significant top-bottom asymmetry appeared, as described in Section 3.1.5. For the plot in Fig. 17, we used PMTs that viewed the source from positions close to perpendicular to the fiberinjection axis of the scintillator ball. As before, the colors reflect the PMT production period: the abscissa contains the angle around this injection axis. For the selected PMTs, the figure shows the following quantity, normalized to the overall average charge:

$$
Q_{o b s}(i) \times R(i)^{2} / a(\theta(i)) /\left(\varepsilon_{q e}(i) \times G(i)\right) .
$$

Here, $\varepsilon_{q e}(i)$ and $G(i)$ are as in Eq. (1); the determinations for each ID-PMT are described in Sections 3.1 .3 and 3.1.5 respectively. The light level used here was equivalent to 30 to 70 photoelectrons at the ID-PMTs. The residual top-bottom asymmetry was $0.8 \%$.
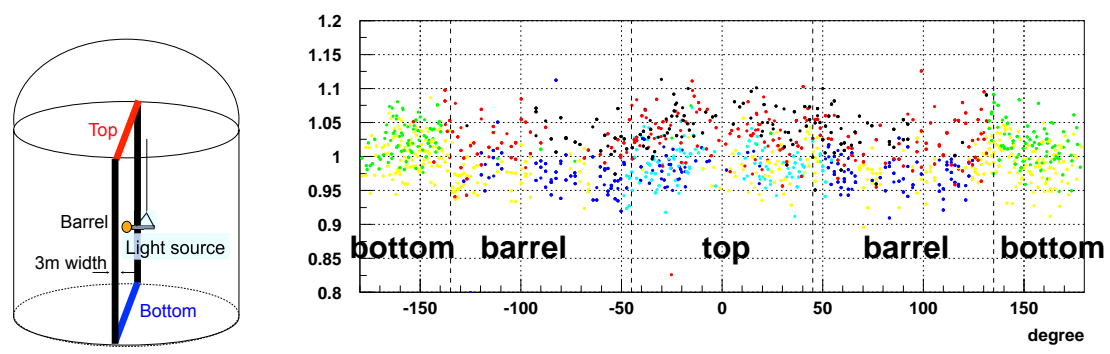

Figure 17: The observed corrected charge divided by the gain and QE factor and normalized by its average over all PMTs. The left side of the figure shows the setup. The horizontal axis in the right figure shows the zenith angle from the top-center point. The color of the points in the right scatter plot corresponds to the PMT production period described in the caption of the Fig. 16

\subsubsection{Linearity of charge measurements}

As discussed in Section 2.2 and Table 2, the new QBEEs have three different ranges for charge digitization. Testing of the on-board QTC chip verified that, within each of these ranges, the linearity of the electronics is better than $1 \%$. However, the overall charge response of the readout system must also include the PMTs. To test the linearity of the whole system, we set up a dedicated calibration run, as illustrated in Fig. 18. The laser system, diffuser ball, and laser monitor PMT are the same as used in the timing calibration (see Section 3.1 .8 for more details).

The goal of this measurement was to cover the widest possible range of light intensities, all the way into saturation. Positioning the diffuser ball in a highly off-center position towards the barrel of the ID resulted in a highly non-uniform light distribution in the detector, and extended the range of intensities seen by the ID-PMTs. To reliably monitor such a wide range of intensities, we deliberately reduced the gains of some ID-PMTs to establish an intensity ladder as the intensity moved through overlapping ranges of good linearity for these PMTs, and their electronics. Ten PMTs in the barrel part of the ID and close to the diffuser ball were selected to monitor the light

${ }^{21}$ October 11,2006 
injected into the tank. These ten PMTs were read out not with the usual QBEE electronics but with a well-understood, high-resolution CAMAC ADC. The ten tubes were set to five different gains, resulting in five monitor ranges with suitable overlap and redundancy. The overall linearity of this intensity monitor ladder and its CAMAC readout electronics was found to be better than $1 \%$.

Thirty different laser intensities were injected into the diffuser ball with the help of a neutraldensity filter wheel. In this way, the electronics recorded the charge output for 30 different, but a priori unknown, light intensities impinging on the ID-PMTs. Generally, those closer to the off-center diffuser ball would be exposed to higher intensities, and those further away would be exposed to lower intensities. The relative intensities for those 30 sets of different-intensity laser shots were established with the help of the ten monitor PMTs. Since we did not want to involve MC estimates for the contributions from scattered and reflected light, we fixed the intensity scale for each individual ID-PMT by choosing the intensity for which its recorded charge was closest to 30 photoelectrons The justification for this value is that the HV settings for each tube were derived from the reference tubes, the HV value of which, in turn, had been established at light level resulting in 30 photoelectrons equivalent charge output. Therefore, the linearity check was anchored at roughly the same intensity as that at which HV settings were established. Using the relative intensities derived from the ten monitor PMTs, we can calculate an expected charge for each of the other 29 intensities that the laser system delivered. We can do that for each ID-PMT, starting from its anchor, i.e. from the intensity at which the charge response of a particular PMT was closest to 30 photoelectrons

Figure 19 shows the result of the linearity measurements. When less or near 200 photoelectrons the response of the ID-PMTs is linear within $1 \%$, and above 1000 photoelectrons the deviation from linearity increases to more than $10 \%$. As indicated earlier, the linearity of the new electronics (QBEEs) is better than $1 \%$ over the whole range. According to the specifications for the 20-inch PMTs, a 5\% anode non-linearity is expected about 250 photoelectrons, and roughly agrees with the measurements. Taking the average over the PMTs contributing to a certain charge range (indicated by red dots in Fig. 19], this non-linearity is considered in MC simulations.

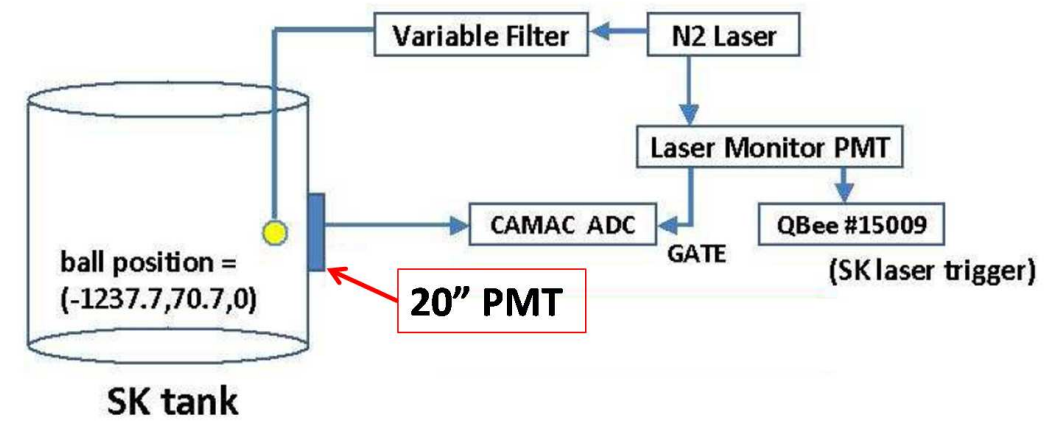

Figure 18: Schematic view of the nonlinearity calibration 


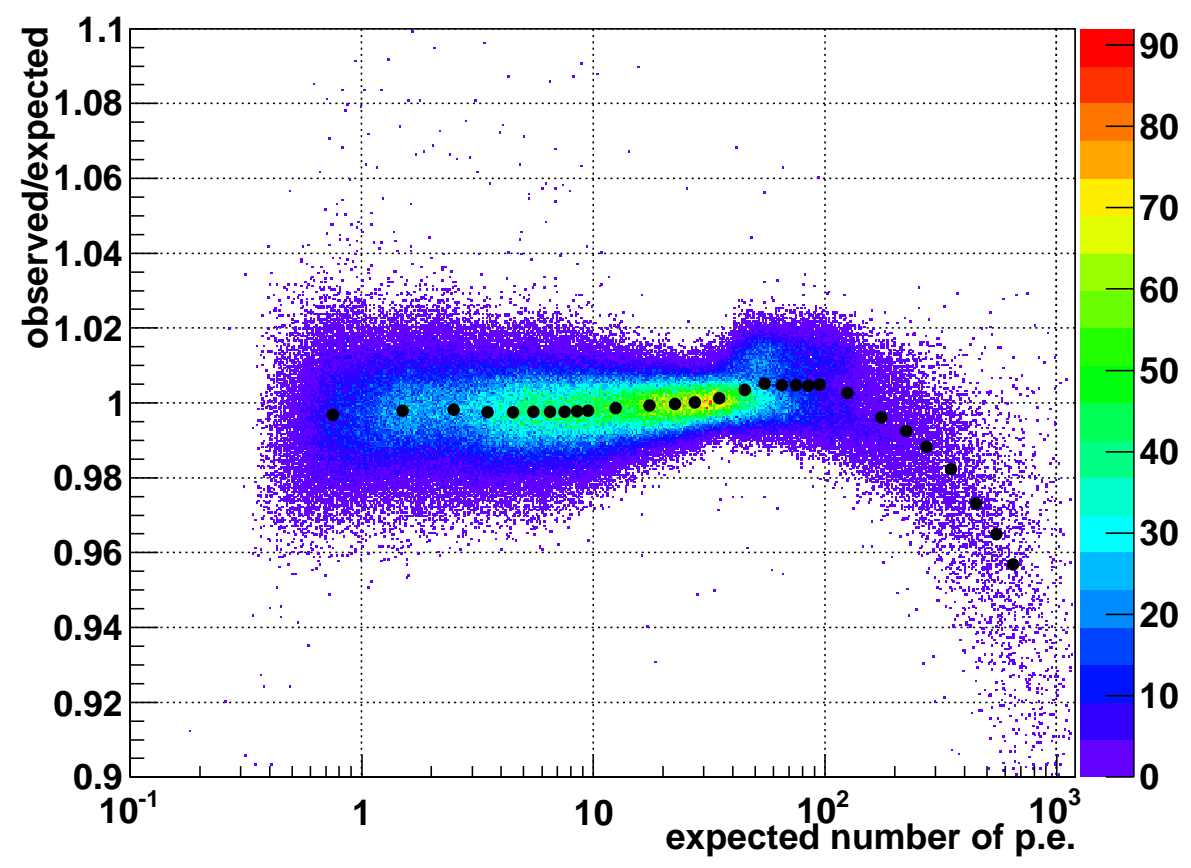

Figure 19: Linearity curve of all the PMTs. The points show the average for each expected number of photoelectrons region. 


\subsubsection{Timing calibration}

The time response of each readout channel, including PMTs and readout electronics, has to be calibrated for precise reconstruction of the event vertices and track directions. The response time of readout channels can vary due to differences of transit time of PMTs, lengths of PMT signal cables, and processing time of readout electronics. In addition, the response time of readout channels (the timing of discriminator output) depends on the pulse heights of PMTs, since the rise time of a large pulse is faster than that of a smaller one. This is known as the "time-walk" effect. The overall process time and the time-walk effect can be calibrated by injecting fast light pulses into PMTs and by varying the intensity of light.

Figure 20 shows a schematic diagram of the SK timing calibration system. The SK uses a

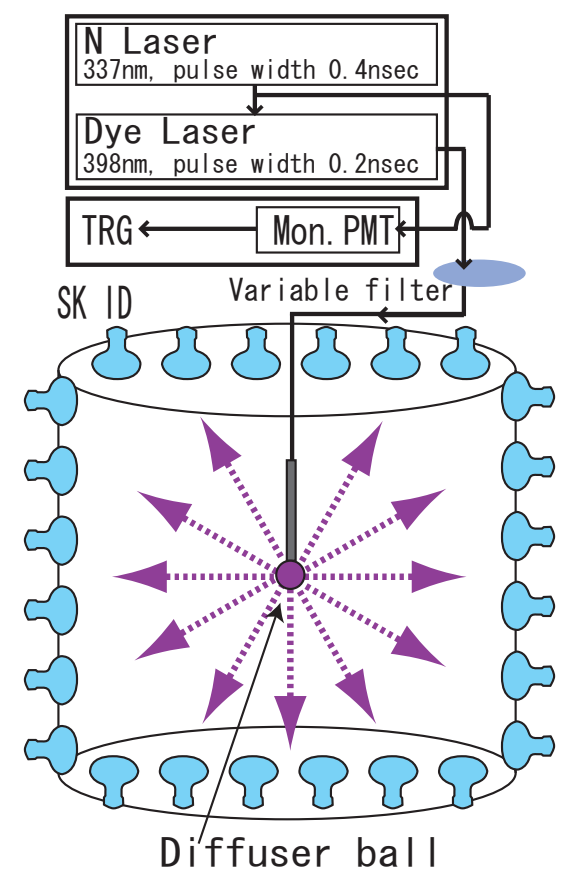

Figure 20: Schematic view of the timing calibration system.

nitrogen laser as a light source for timing calibrations. The nitrogen laser (USHO KEC-100) is a gas flow laser that emits fast pulsing light of $0.4 \mathrm{~ns}$ FWHM at a wavelength of $337 \mathrm{~nm}$. The laser output is monitored by a fast response 2-inch PMT (Hamamatsu H2431-50, rise time: 0.7 ns, T.T.S: $0.37 \mathrm{~ns}$ ). This monitor PMT is used to define the time of laser light injection. The wavelength of the laser light is shifted to $398 \mathrm{~nm}$ by a dye, where the convoluted response with Cherenkov spectrum, light absorption spectrum and quantum efficiency of the PMTs is almost maximum. The pulse width of the dye is $0.2 \mathrm{~ns}$ FWHM. The light intensity is varied by a variable optical filter. We use the optical filter to measure the time responses of readout channels at various pulse height. The filtered light is guided into the detector by an optical fiber $(400 \mu \mathrm{m}$ core) and injected into a diffuser ball located near the center of the tank to produce an isotropic light. Figure 21 shows a cross section of the diffuser ball. Directional variations in the photon 


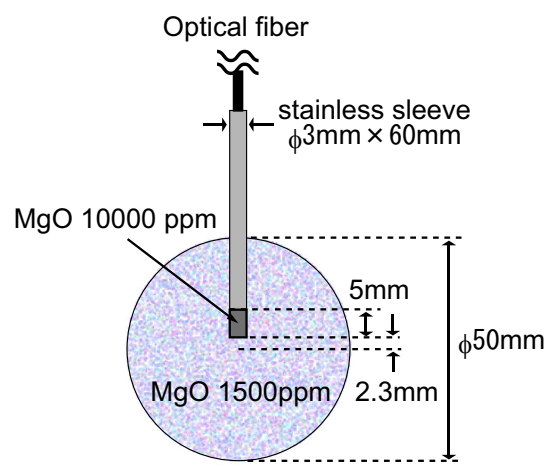

Figure 21: Cross section of the diffuser ball.

emission time of the diffuser ball were measured to be less than $0.2 \mathrm{~ns}$.

Timing calibrations for SK ID readout channels were conducted based on two-dimensional, timing versus pulse height (charge), correlation tables that are called "TQ distributions". Figure 22 shows a typical scatter plot of the TQ distribution for one readout channel. The calibration constants, called the "TQ-map", are derived by fitting the TQ distribution to polynomial functions. A TQ-map includes overall process time and the time-walk effect; each readout channel has its own TQ-map.

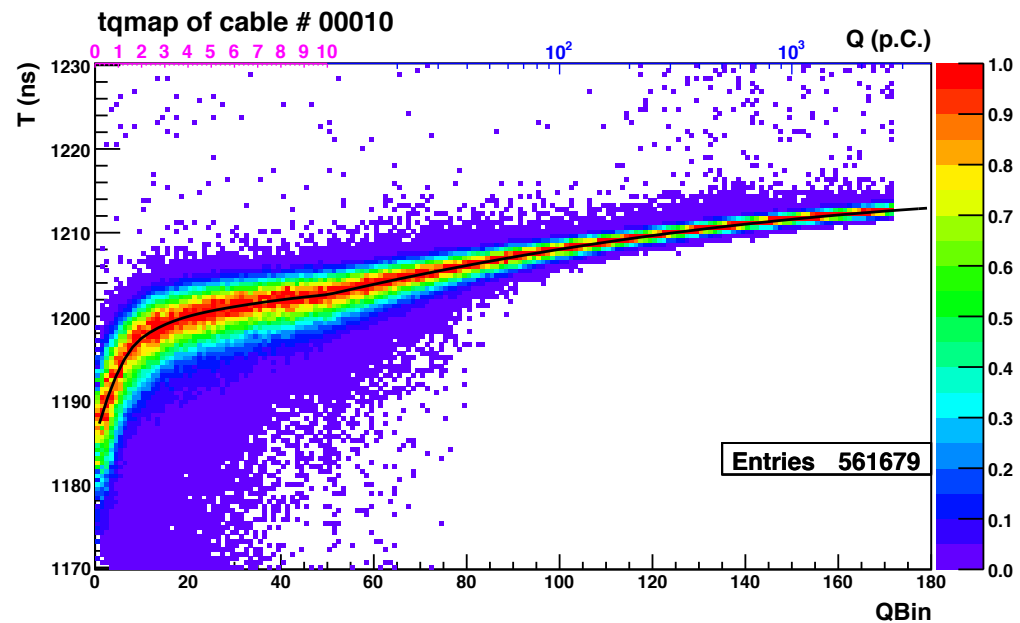

Figure 22: Typical TQ distribution for a readout channel. The horizontal axis is charge (Qbin) of each hit, and the vertical axis is time-of-flight-corrected timing (T) of the hits. Larger (smaller) T corresponds to earlier (later) hits in this figure.

In the laser event selection, we require that the monitor PMT is fired. The fired timing defines the reference timing of the laser flashed. For laser events, we apply a timing correction, called time-of-flight (TOF) correction, that subtracts time of flight from the diffuser ball to the respective PMT position using group velocity of light with the measured wavelength, $\sim 398 \mathrm{~nm}$. Using 
the TOF-corrected hit timing, "laser hits" are defined as hits in a time window $\pm 50 \mathrm{~ns}$ around the hit timing of the monitor PMT.

The selected laser hits of each readout channel are divided into 180 bins of charge, called "Qbin"s. Each Qbin is defined as the amount of charge from the PMT in pC; they are defined on a linear scale from 0 to $10 \mathrm{pC}(0.2 \mathrm{pC} / \mathrm{Qbin})$ and on a logarithmic scale from 10 to $3981 \mathrm{pC}$ $(50 \log (\mathrm{pC}) / \mathrm{Qbin})$. After the TQ distributions are divided into 180 Qbins, the timing distribution is smoothed by a Gaussian to minimize statistical fluctuations. Although the timing distribution in each Qbin is almost Gaussian, the timing distributions have a small asymmetric feature because of an asymmetric time response of PMT and contributions from direct and indirect light; direct light causes early hits, while indirect light causes late hits due to reflection and scattering. In order to take the asymmetric feature into account, the timing distribution in each Qbin is fitted to an asymmetric Gaussian, which provides the peak timing and standard deviation. The peak timing and standard deviations for respective charges are fitted by polynomial functions depending on Qbin:

$$
\begin{aligned}
\operatorname{polN}(x) & \equiv p_{0}+p_{1} x+p_{2} x^{2}+\ldots+p_{N} x^{N} \\
\text { Qbin } \leq 10: F_{1}(x) & \equiv \operatorname{pol} 3(x), \\
\text { Qbin } \leq 50: F_{2}(x) & \equiv F_{1}(10)+(x-10) \cdot\left[F_{1}^{\prime}(10)+(x-10) \cdot \operatorname{pol} 3(x-10)\right], \\
\text { Qbin }>50: F_{3}(x) & \equiv F_{2}(50)+(x-50) \cdot \operatorname{pol} 6(x-50) .
\end{aligned}
$$

where $F_{1}^{\prime}$ in Eq. 87 is a derivation of $F_{1}$, that is introduced for continuity between $F_{1}(x)$ and $F_{2}(x)$ at Qbin $=10$. In Eq. (8) and Eq. (9), $F_{1}(10)$ and $F_{2}(50)$ are introduced to satisfy the boundary conditions to connect $F_{i}(x)(i=1,2,3)$ at Qbin $=10$ and Qbin $=50 . F_{1}(x)$ and $F_{2}(x)$ have 4 fit parameters each, and 7 fit parameters in $F_{3}(x)$. Thus, the number of fit parameter is 15 in total. The parameters resulting from the fit are saved in a database as the TQ-map and are used to correct the time response of each readout channel.

The timing resolution of the SK detector is evaluated using the same data set as used for the TQ-map evaluation. To evaluate the timing resolution, all PMT timing distributions, corrected by their TQ-maps, are accumulated in each Qbin and the timing distributions in Qbins are fitted by an asymmetric Gaussian that is defined,

$$
\begin{aligned}
& f\left(t ; t>T_{\text {peak }}\right) \equiv A_{1} \cdot \exp \left(-\left(t-T_{\text {peak }}\right)^{2} / \sigma_{t}^{2}\right)+B_{1}, \\
& f\left(t ; t \leq T_{\text {peak }}\right) \equiv A_{2} \cdot \exp \left(-\left(t-T_{\text {peak }}\right)^{2} / \sigma_{t}^{\prime 2}\right)+B_{2},
\end{aligned}
$$

where $A_{i}, B_{i}(i=1,2), \sigma_{t}$ and $\sigma_{t}^{\prime}$ are fit parameters (note that, in these equations, a larger $t$ corresponds to earlier hits). The fit parameters need to satisfy a boundary condition, $A_{1}+B_{1}=$ $A_{2}+B_{2}$, to connect two Gaussian functions at $t=T_{\text {peak }}$. As an example, Fig. 23 shows the timing distribution and the function resulting from the fit for Qbin $=14$.

Figure 24 shows the timing resolution, i.e. the standard deviation $\sigma_{t}$ and $\sigma_{t}^{\prime}$, as a function of charge. They are implemented in the SK detector simulation. By implementing both $\sigma_{t}$ and $\sigma_{t}^{\prime}$, SK detector simulation reproduces timing distributions of data, e.g. LINAC calibration ${ }^{22}$ data, better than by implementing only $\sigma_{t}$.

The task of real-time monitoring is to monitor the deviation of the time-response of readout channels during SK detector operation, and to apply a correction to the TQ-map as necessary.

For the real-time monitoring of the time response of readout channels, SK employs a nitrogen laser (Laser Science Instruments, VSL-337 and DLM-120 dye laser system). This laser system

${ }^{22}$ The details of SK LINAC calibration can be found in reference [1]. 


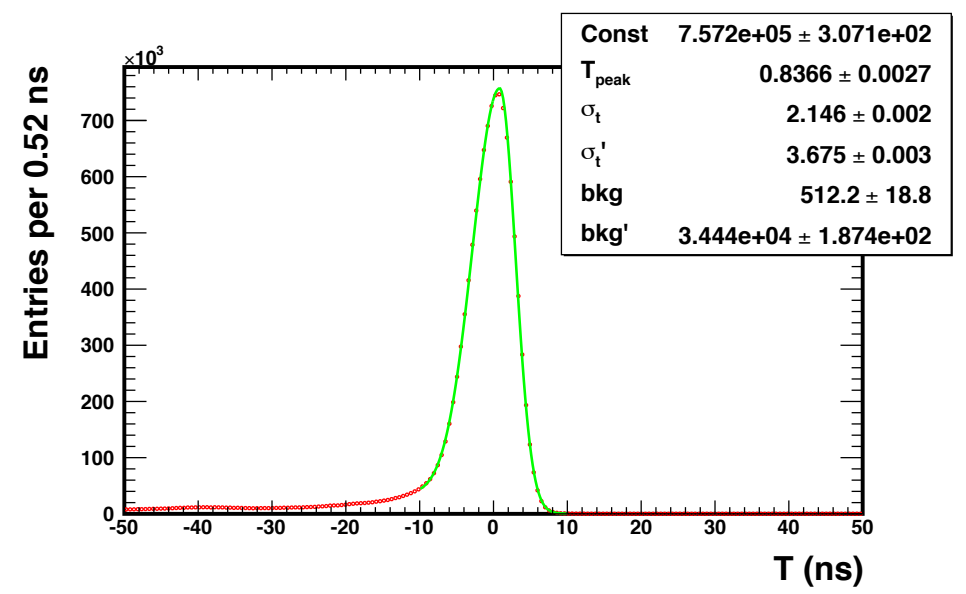

Figure 23: Timing distribution added over all the readout channels in Qbin $=14(\sim 1$ photoelectron $)$. The result of the fit to an asymmetric Gaussian is shown by the solid curve.

uses sealed nitrogen as a gain medium and is better suited for continuous operation than the gasflow laser that used for the TQ-map evaluation. This laser also emits fast pulsing light of less than $4 \mathrm{~ns}$ FWHM, and the wavelength of the light is $337.1 \mathrm{~nm}$. The wavelength of the laser light is shifted by a dye system to $398 \mathrm{~nm}$. The light output of the dye system is injected into the same diffuser ball as used for TQ-map evaluation.

For real-time monitoring, SK uses a fixed light intensity that is adjusted to obtain an averaged SK ID occupancy of $\sim 99 \%$ and an averaged charge of $\sim 20$ photoelectrons per readout channel at maximum. The frequency of the flashing laser light is $\sim 0.03 \mathrm{~Hz}$, and a typical 24-hour operation provides a statistical accuracy of $\sim 0.05 \mathrm{~ns}$ for monitoring the time response of the readout channels.

Figure 25 shows timing-offset values as a function of time for a typical readout channel; here, the timing offset is defined as the difference between the measured time response from real-time monitoring and that from pre-defined TQ-map. As shown in the figure, the time responses of readout channels have been stable within \pm 0.1 ns for a few years.

\subsection{Calibration for photon tracking}

In this section, we summarize results from measurements of water quality and light reflection at the ID wall used in SK detector simulations (SK-MC).

\subsubsection{Light absorption and scattering in water}

By injecting a collimated laser beam into the SK tank (Fig. 26) and comparing the timing and spatial distributions of the light with MC, we can extract light absorption and scattering coefficients as functions of wavelength. The details of the experimental setup and data analysis are described in Section 8.2 [1]. Figure 27 compares data and MC results for typical hit PMT time distributions after time-of-flight (TOF based on the distance from the target of the laser at the bottom region to the hit PMT position). The left region is from scattered photons, while the right peak represents photons reflected by the bottom PMTs and black sheets. The total number of scattered photons and the shape of the time distribution are used to tune the symmetric and 


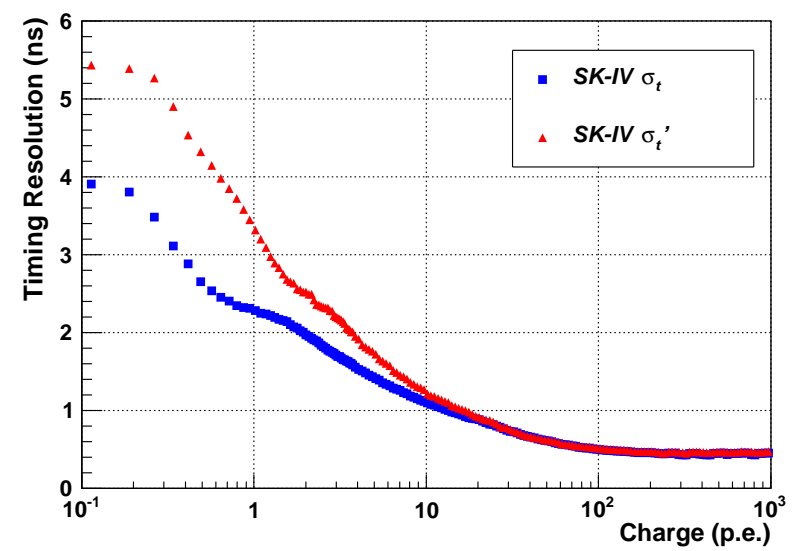

Figure 24: Timing resolution $\sigma_{t}$ (square) and $\sigma_{t}^{\prime}$ (triangle) as a function of charge (photoelectron) for SK-IV. 


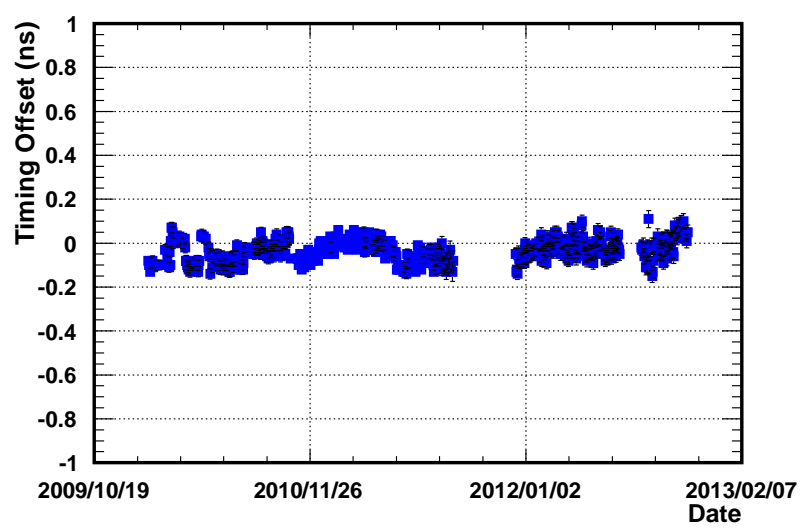

Figure 25: Timing-offset values with respect to pre-defined TQ-map for a typical readout channel as a function of time. The error bar on each point indicates statistical uncertainty.

asymmetric scattering and the absorption parameters for the SK-MC. We generate various timing distributions with different MC parameters and select the one that minimizes the $\chi^{2}$ value for the difference between data and $\mathrm{MC}$ results.

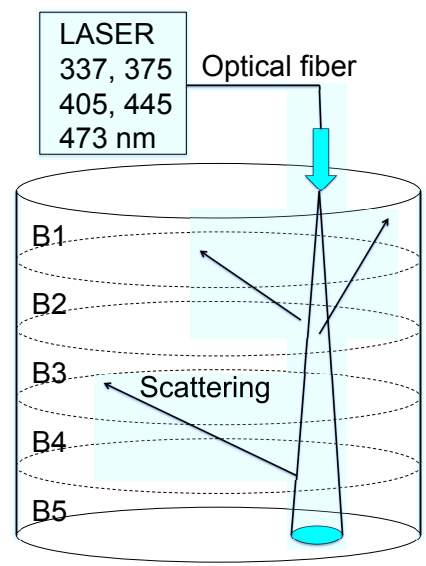

Figure 26: Real-time laser system for measurements of absorption and scattering of the Cherenkov light in water and reflectivity at the PMT surface. Analysis was performed using PMTs belonging to five divisions of the barrel region, B1 to B5, and top. The blue shaded circle spot at the bottom region indicates the beam target used in the TOF calculation.

The number of photons with a wavelength $(\lambda)$ in water decreases gradually according to:

$$
I(\lambda)=I_{0}(\lambda) e^{-\frac{l}{L(\lambda)}}
$$




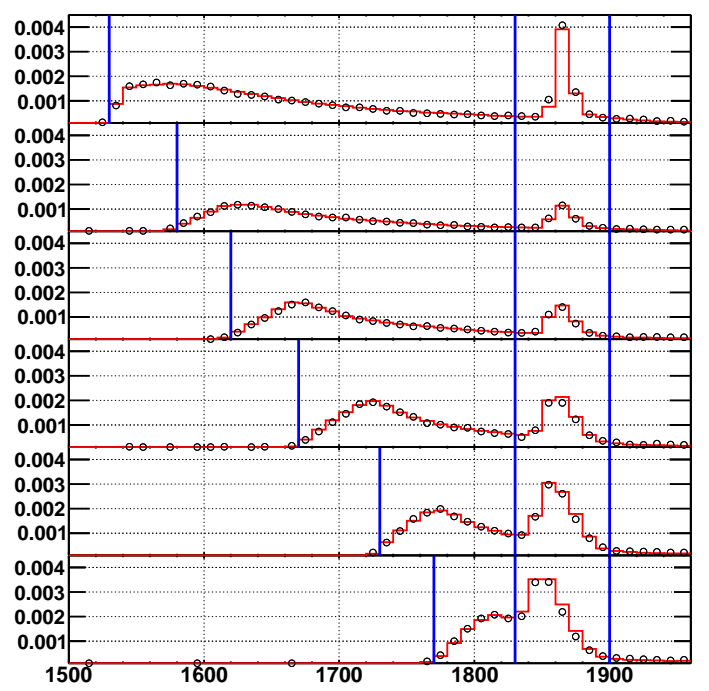

Figure 27: Typical TOF-subtracted (see text) timing distributions for the vertical down-going laser beam with the wavelength at $405 \mathrm{~nm}$ between data (black circle) and MC (red histogram, the best tune) normalized by observed total photoelectrons. The top plot is for the PMTs at the SK tank top wall. The second to bottom plots correspond to the five barrel wall regions from top to bottom of the SK tank as indicated in Fig. 26 The time region between the left two blue solid vertical lines is used to measure the absorption and scattering described in this Section 3.2.1 and the right region is used for the measurement of reflection described in Section 3.2.2

where $l$ is the travel length of the light, $I_{0}(\lambda)$ is the initial intensity, $I(\lambda)$ is the intensity at $l$, and $L(\lambda)$ is the total attenuation length caused by scattering and absorption, which we call water transparency. $L(\lambda)$ is defined in the SK-MC as follows:

$$
L(\lambda)=\frac{1}{\alpha_{a b s}(\lambda)+\alpha_{s y m}(\lambda)+\alpha_{a s y}(\lambda)},
$$

where $\alpha_{a b s}(\lambda), \alpha_{\text {sym }}(\lambda)$, and $\alpha_{\text {asy }}(\lambda)$ are described below. These are tuning parameters used in the SK-MC, they are SK-based empirical functions and do not exactly represent real physical properties.

The absorption amplitude $\alpha_{a b s}\left(\mathrm{~m}^{-1}\right)$ as a function of wavelength $\lambda(\mathrm{nm})$ is empirically determined using the laser beam data in

$$
\alpha_{a b s}(\lambda)=P_{0} \times \frac{P_{1}}{\lambda^{4}}+C,
$$

where the second term $C$ is the amplitude based on the experimental data for $\lambda \geq 464 \mathrm{~nm}$ [17, 18], while the following formula

$$
C=P_{0} \times P_{2} \times(\lambda / 500)^{P_{3}}
$$

is used for $\lambda \leq 464 \mathrm{~nm}$. Values for parameters $P_{0}-P_{3}$ are obtained from a fit to the data. The results of the fit of Eq. 14 and () to the data of April 2009 are shown in Fig. 28.

The angular distribution of scattered light is divided into two components, "symmetric" (described by $1+\cos ^{2} \theta$ where $\theta$ is the scattered photon angle) and "asymmetric" for which the scattering probability increases linearly from $\cos \theta=0$ to 1 , and no scattering occurs for $\cos \theta<0$ ). 


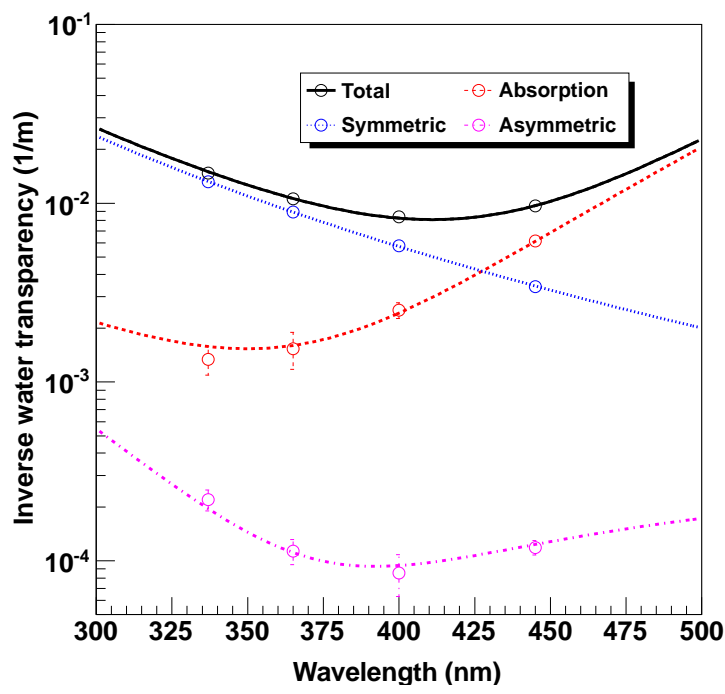

Figure 28: Typical fitted water coefficient functions used in the SK-MC. The points are the data obtained in April 2009. The each lines through the points for absorption, symmetric scattering and asymmetric scattering show the fitted functions while the top line shows the total of all fitted functions added together. 


\begin{tabular}{|cccc|cc|ccc|}
\hline$P_{0}$ & $P_{1}$ & $P_{2}$ & $P_{3}$ & $P_{4}$ & $P_{5}$ & $P_{6}$ & $P_{7}$ & $P_{8}$ \\
\hline 0.624 & $2.96 \times 10^{7}$ & $3.24 \times 10^{-2}$ & 10.9 & $8.51 \times 10^{7}$ & $1.14 \times 10^{5}$ & $1.00 \times 10^{-4}$ & $4.62 \times 10^{6}$ & 392 \\
\hline
\end{tabular}

Table 3: Summary of the typical fitted water quality parameters in Eq. 14, (), 16, and (17) obtained from April 2009 data.

The "symmetric" scattering consists of Rayleigh and symmetric Mie scattering, while the "asymmetric" scattering consists of forward Mie scattering. The Mie scattering typically has a forwardpeaked distribution for particles whose size is greater than wavelength of the light. The "asymmetric" scattered angle function is a simple approximation because details of the particle sizes are unknown. The "symmetric" $\alpha_{\text {sym }}$ and "asymmetric" $\alpha_{a s y m}$ scattering amplitudes are empirically determined from

$$
\begin{gathered}
\alpha_{\text {sym }}(\lambda)=\frac{P_{4}}{\lambda^{4}} \times\left(1.0+\frac{P_{5}}{\lambda^{2}}\right), \\
\alpha_{\text {asym }}(\lambda)=P_{6} \times\left(1.0+\frac{P_{7}}{\lambda^{4}} \times\left(\lambda-P_{8}\right)^{2}\right),
\end{gathered}
$$

where $P_{4}-P_{8}$ are fitting parameters. Figure 28 shows a typical result of the scattering fit to Eq. (16) and (17).

Table 3 gives typical values of the fitted parameters determined from the laser beam data. Using the values in Table 3 , the values for $1 / \alpha_{a b s}, 1 / \alpha_{\text {sym }}$, and $1 / \alpha_{\text {asym }}$ are calculated to be $4.02 \times 10^{2} \mathrm{~m}, 1.76 \times 10^{2} \mathrm{~m}$, and $9.89 \times 10^{3} \mathrm{~m}$, respectively. These lead to $L \sim 120 \mathrm{~m}$ at $\lambda=$ $400 \mathrm{~nm}$.

Obtaining real-time laser-beam data continues and time variation of each coefficient are monitored at several wavelengths. Figure 29 shows time variations in absorption and scattering in SK-IV. Each parameter was determined by applying the same analysis ( $\chi^{2}$ method) on the real data from each laser run. The symmetric scattering coefficient is relatively stable (the RMS/mean was about 3\% August 2008 - November 2012). Both the absorption and asymmetric scattering coefficients have relatively larger time dependence (about $20 \%-40 \%$ and $20 \%-60 \%$ in the same time period, respectively). Since asymmetric scattering is one order of magnitude smaller than absorption, the time variations in water transparency are mainly caused by absorption.

As described in Section 2.4, water quality has position dependence. The vertical dependence is determined from the nickel data obtained every month and monitored with the real-time Xe system as described in Section 3.1.2. A similar $z$-dependence in the water quality is seen in other control sample data, such as decay electrons from cosmic-ray stopping muons. Figure 30 shows the top-bottom asymmetry of the hit probability normalized by the average of all the PMTs as a function of time. The top-bottom asymmetry $\left(\alpha_{t b a}\right)$ is defined by

$$
\alpha_{\text {tba }}=\left(\left\langle N_{\text {top }}\right\rangle-\left\langle N_{\text {bottom }}\right\rangle\right) /\left\langle N_{\text {barrel }}\right\rangle
$$

where $\left\langle N_{\text {top }}\right\rangle,\left\langle N_{\text {bottom }}\right\rangle$ and $\left\langle N_{\text {barrel }}\right\rangle$ are the averages of the hit probabilities of top, bottom and barrel of SK, respectively. The average of the hit probability on the top PMTs is about 5\% smaller than that on the bottom PMTs, as discussed in Section 2.4. The nickel and Xe measurements are in accordance over the entire SK-IV time period. Since the time variation is mainly caused by absorption, as described above, the vertical position and time dependence of the water quality is introduced in SK-MC by multiplying $\alpha_{a b s}$ by a factor $A(z, t)$ in Eq. (13): 


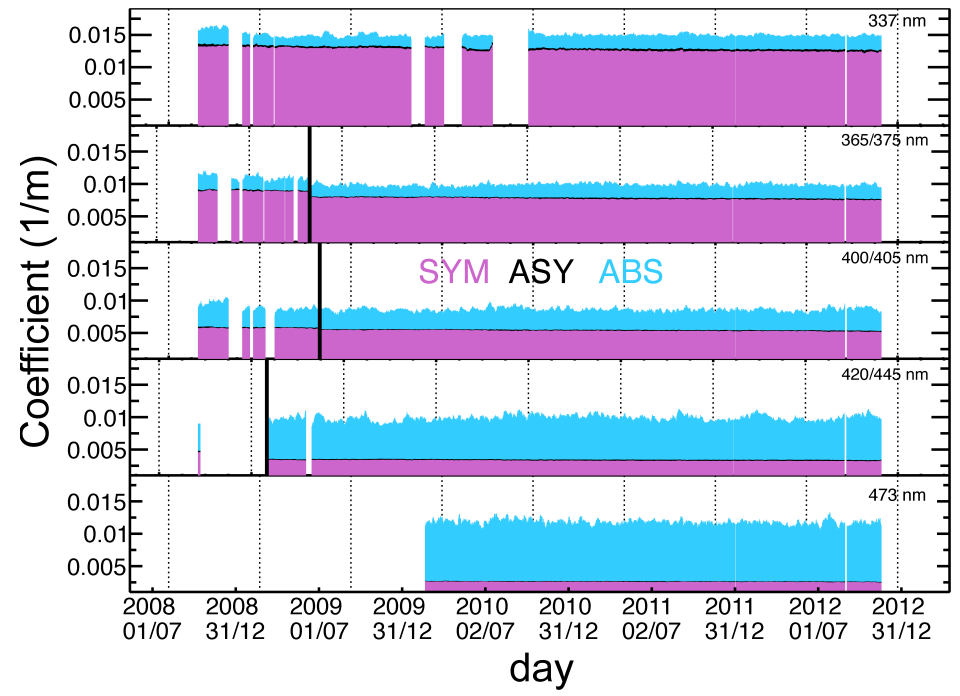

Figure 29: Time variation of the water parameters in SK-IV from October 2008 to November 2012. The vertical axis is the inverse water transparency $\left[\mathrm{m}^{-1}\right]$. The absorption, asymmetric scattering, and symmetric scattering for each wavelength are shown in blue (top), black (middle), and purple (bottom), respectively. Note that the asymmetric scattering (black) is smaller than others by one order of magnitude. The wavelength was changed in 2008 from $365 \mathrm{~nm}$ to $375 \mathrm{~nm}$, from $400 \mathrm{~nm}$ to $405 \mathrm{~nm}$, and from $420 \mathrm{~nm}$ to $445 \mathrm{~nm}$ as indicated by the black vertical bars.

$$
\begin{aligned}
A(z, t) & =1+z \cdot \beta(t) \quad \text { for } z \geq-11 \mathrm{~m} \\
& =1-11 \cdot \beta(t) \quad \text { for } z \leq-11 \mathrm{~m}
\end{aligned}
$$

As shown in Fig. 4), the value of $A(z, t)$ is assumed to be constant below $z=-11 \mathrm{~m}$ where water convection from the pure water inlet at the tank bottom is relatively stable and effective, while we assume that absorption changes linearly for $z>-11 \mathrm{~m}$. To determine the slope $(\beta)$, the topbottom asymmetry of the hit probability $\left(\alpha_{t b a}\right)$ in the nickel data was compared with various MC samples with different values of $\beta$ in the nickel data analysis. We found the following relationship between $\beta$ and $\alpha_{t b a}(\%)$;

$$
\beta(1 / m)=\left(-0.163 \times\left(\alpha_{t b a}\right)^{2}-3.676 \times \alpha_{t b a}\right) \times 10^{-3}
$$

For example, in April $2009 \alpha_{t b a}$ and $\beta$ are $-4.91 \%$ and 0.01 , respectively.

\subsubsection{Light reflection at PMT and black sheet}

Light reflection at the PMT surface is tuned using the same laser data used to determine the water quality parameters, as described in Section 3.2.1. Typical time distributions for data and MC after simulation tuning are shown in Fig. 27. Four layers of material (refractive index) from the surface to the inside of the PMT are taken into account; water (1.33), glass $\left(1.472+3670 / \lambda^{2}\right.$, where $\lambda$ is the wavelength in $\mathrm{nm})$, bialkali $\left(n_{\text {real }}+i \cdot n_{\text {img }}\right)$ [20], and vacuum (1.0). Here, $n_{\text {real }}$ and $n_{\text {img }}$ are the real and imaginary parts of the complex refractive index, and an appropriate thickness of the photocathode was based on information from the manufacturer [22]. The best fit 


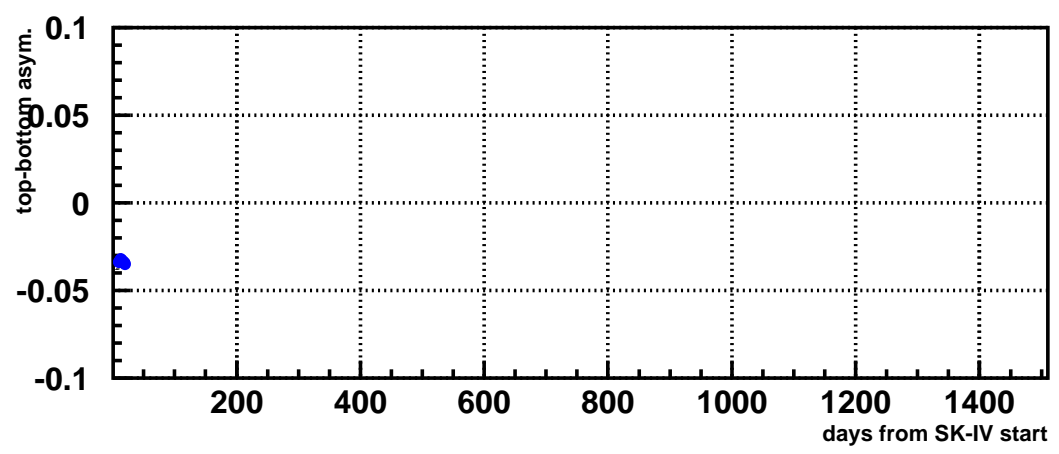

Figure 30: Top-bottom hit probability asymmetry as a function of time for SK-IV. The vertical axis gives the value obtained using the averaged hit probability in top, bottom and barrel PMTs defined as Eq. (18). The red points show the nickel data and the blue thick curve shows the real-time Xe data described in Section 3.1 .2

values from the tuning were: $n_{\text {img }} 1.667$ and $n_{\text {real }} 2.31,2.69,3.06$, and 3.24 at $\lambda=337,365,400$ and $420 \mathrm{~nm}$, respectively. Note that these fitted values include the effects from several materials inside of the PMT as well as the photocathode.

Cherenkov photons are reflected or absorbed on the black sheet. The reflectivity of the black sheet used in SK-MC is measured by a light injector set in the SK tank. The experimental setup is shown in Fig. 31 The reflected charge $\left(Q_{\text {scattered }}\right)$ was measured at three incident angles $\left(30^{\circ}\right.$, $45^{\circ}$, and $\left.60^{\circ}\right)$ with three wavelengths $(337 \mathrm{~nm}, 400 \mathrm{~nm}$, and $420 \mathrm{~nm})$. For reference, the direct charges $\left(Q_{\text {direct }}\right)$ without the black sheet were also measured. The total reflectivity is tuned using the ratio $R=Q_{\text {scattered }} / Q_{\text {direct }}$. The tuning results are shown in Fig. 32 The adjustment resulted in agreement between data and MC at better than the $\pm 1 \%$ level at each wavelength and position.

\section{Outer detector calibration}

\subsection{Introduction}

The SK-I OD is described in [1]. The OD was completely rebuilt to its original configuration for SK-II. For SK-III, dead phototubes were replaced and vertical sheets of Tyvek were installed to optically separate the barrel and endcap regions as shown in Fig. 33 The optical segmentation was implemented to enhance rejection of background "corner-clipping" cosmic-ray muons from neutrino interactions with an exiting lepton, e.g. partially-contained (PC) events used in atmospheric neutrino analyses [7, 5].

For SK-IV, the electronics were completely replaced [19] to use the same system as the ID (the previous custom electronics are described in [1]). Because the new electronics were more sensitive to cross-talk between the ID and the OD, the OD tube gains were reduced for SK-IV and the calibrations were redone. The OD calibrations used for SK-IV physics data analyses are summarized here.

The primary role of the OD is to identify incoming cosmic rays and atmospheric neutrino interactions with particles leaving the ID. It also serves as a passive buffer against low-energy 

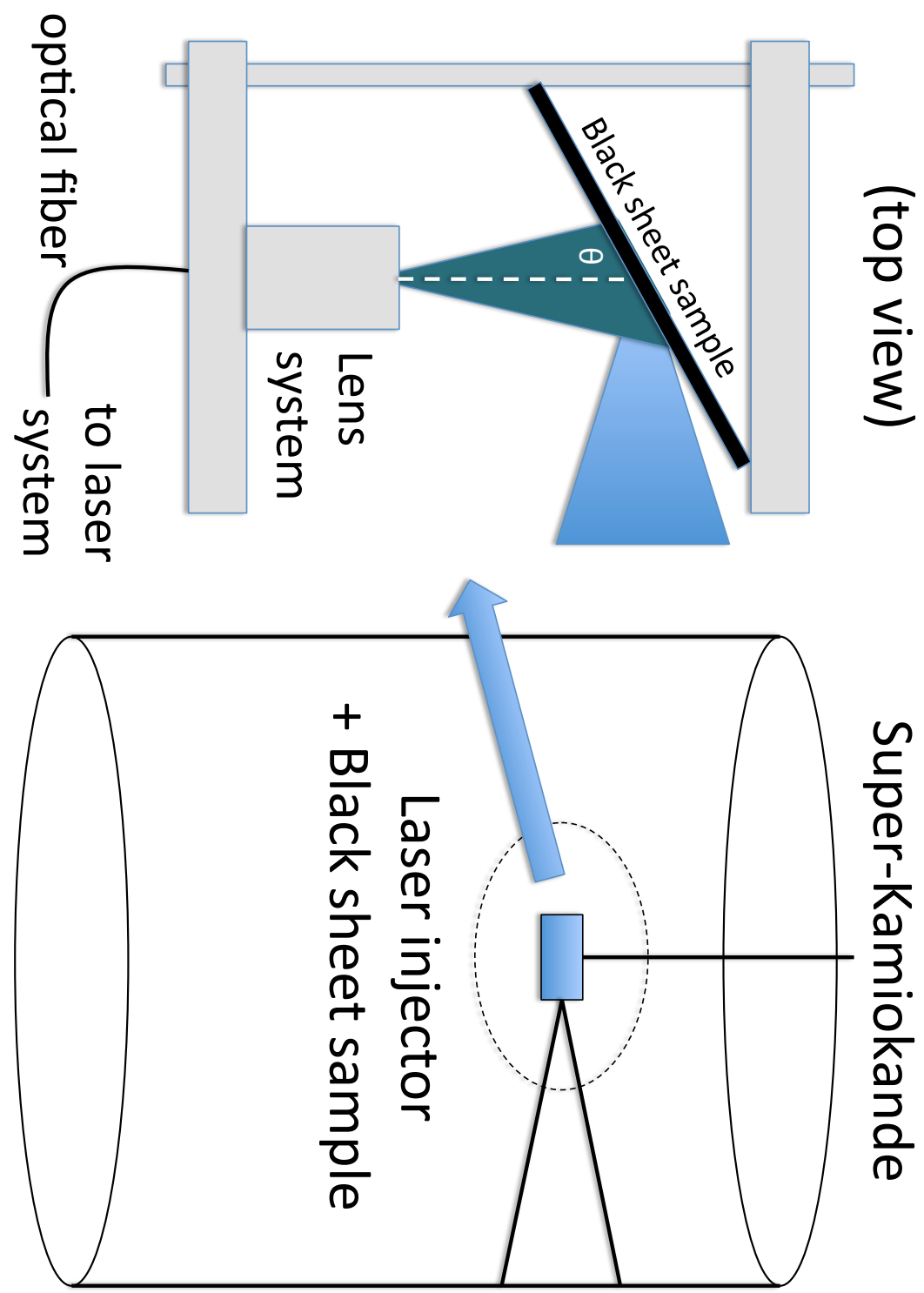

Figure 31: Schematic view of a laser light injector for the reflectivity measurement of a black sheet. The left figure shows the top view. The injector was inserted to the center part of the SK tank and the reflected light was measured by the SK ID-PMTs. For the normalization of reflectivity, data were taken without the black sheet. 

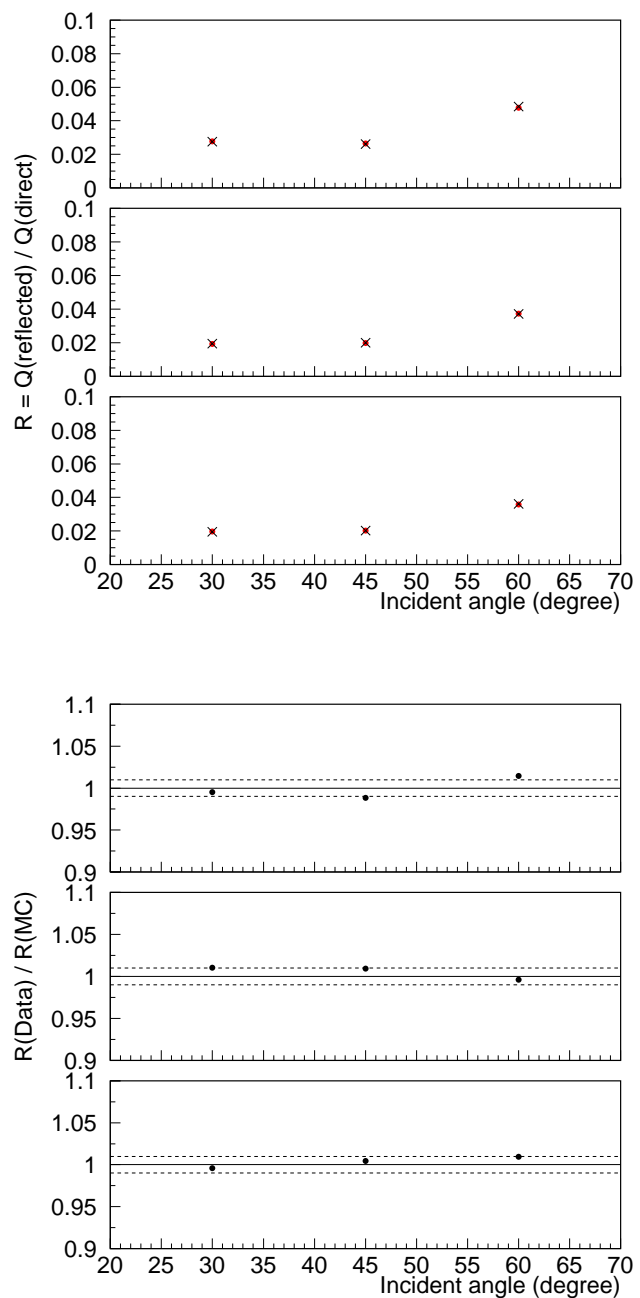

Figure 32: The result of black sheet reflectivity tuning. The top figure is for the wavelength at $337 \mathrm{~nm}$, the middle is for $400 \mathrm{~nm}$ and the bottom is for $420 \mathrm{~nm}$. The left figure shows the charge ratio (see text) of data (black crosses) and MC (red circles) as a function of incident angle. The right figure shows the ratio between data and $\mathrm{MC}$ for the the left plots. 


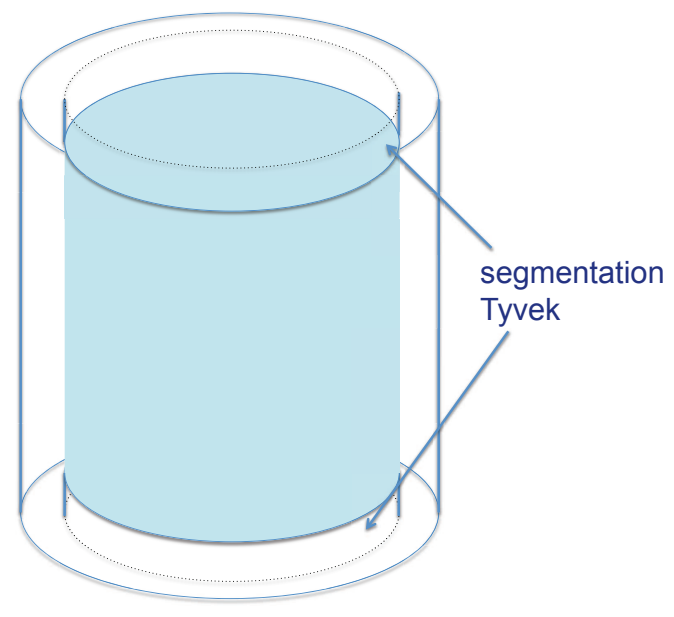

Figure 33: Schematic showing the OD segmentation Tyvek barrier to optically separate the endcap and barrel regions of the $\mathrm{OD}$.

particles (gamma rays and fast neutrons) from the surrounding rock. The OD has been used for rough reconstruction of very high-energy events (above TeV) that saturate the ID [13]. The OD is also important to separate PC stopping and through-going events used for atmospheric neutrino oscillation analyses [7, 5]. It is also used for selecting T2K beam neutrino events with light in the OD [21]. Requirements for OD calibration are not as stringent as for the ID. OD charge reconstruction accuracy of about $10 \%-20 \%$ and timing to 5-10 ns are generally sufficient for SK physics needs. The calibration and simulation parameter tuning for the OD-PMTs are done differently than for the ID. OD time and charge calibrations are done using cosmic-ray and dark rate data together with the nitrogen-dye laser that is used for the ID timing calibrations described in Section 3.1 .8

\subsection{OD-PMTS}

There are 1885 8-inch PMTs installed in the OD, they are mounted on the outside of the steel structure that divides the inner and outer detectors. Each is installed with a wavelength-shifting plate [1]. For SK-I, the PMTs were Hamamatsu R1408 recycled from the IMB detector. For SKII, some new Hamamatsu R5912 PMTs were installed. The new tubes were installed primarily on the bottom part of the detector, while the older ones were installed towards the upper part of the detector where the water pressure is lower. For the SK-III upgrade, dead OD tubes were replaced. For SK-III and IV there are 1293 new and 591 old PMTs in the OD.

For SK-IV, high-voltages applied to the OD-PMTs were reduced in order to reduce cross-talk with the ID, to which the new electronics are sensitive. The applied high-voltages of the new OD-PMTs were lowered by $400 \mathrm{~V}$ and those of the old OD-PMTs were lowered by $200 \mathrm{~V}$. This change reduced the gains to about $1 / 5$ and $1 / 3$ of the original gains for the new and old tubes, 
respectively. New and old tubes have different single-pe characteristics, with new tubes showing generally clear single-pe peaks and old tubes having approximately exponential single-pe charge distributions. Figure 34 shows typical single-pe distributions for new and old OD-PMTs. At the same time as the gain change, the QBEE charge threshold was changed from about $-25 \mathrm{mV}$ to about $-1.4 \mathrm{mV}$, in order to maintain efficiency for single-pe detection. Using the lower-intensity laser described in Section 4.3 we experimentally confirmed that we do not lose single-pe hit efficiency for SK-IV as compared to SK-III.
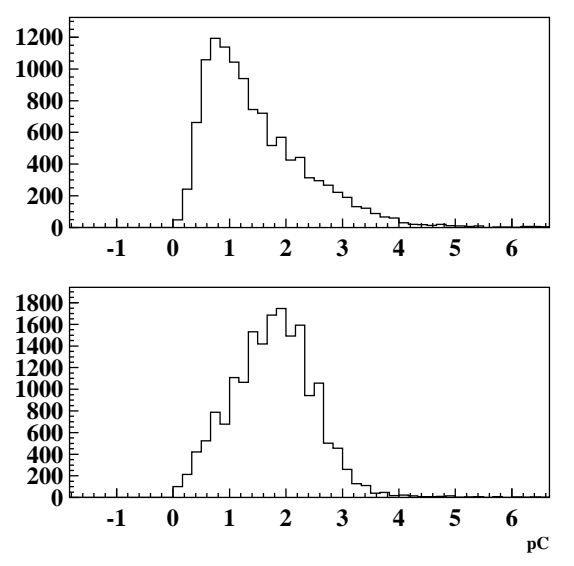

Figure 34: OD-PMT single-pe distributions, in units of pC. Top: an example old (IMB) tube, showing roughly exponential charge distribution. Bottom: an example new tube, showing a clear single-pe peak.

\subsection{OD calibration hardware}

The laser output is sent to 52 optical fibers that are routed to the OD. The fibers are of UV-VIS grade fused silica: each has a $200 \mu \mathrm{m}$ diameter core, a $10 \mu \mathrm{m}$ fused silica cladding and a 10 $\mu \mathrm{m}$ protective layer. The fibers are fed through a hole near the middle of the top of the tank, routed to locations around the $\mathrm{OD}$, and have their ends mounted to the outer walls to illuminate the OD-PMTs. The OD fiber ends are mounted on white Tyvek-wrapped backboards clamped to structural bolts on the SK water tank. The fiber coordinates are shown in Fig. 35 A total of 14 fibers are installed on the top of the tank, 12 are installed on the walls at about $z=980 \mathrm{~cm}$ in tank coordinates, 12 are installed on the walls at about $z=-1017 \mathrm{~cm}$, and 14 are installed on the bottom. The fibers are $72 \mathrm{~m}$ long except for those going to the bottom of the tank, that are $110 \mathrm{~m}$ long. Light from the fiber ends is diffused by a mixture of optical cement and titanium dioxide applied to the ends.

\subsection{OD charge calibration}

\subsubsection{OD linear charge response}

To determine the charge in $\mathrm{pC}$ corresponding to a single-pe in SK-IV, we use a noninvasive "dark rate method" for charge calibration based on OD hits outside the trigger time window. Hits preceding the trigger time have high probability of being single-pe hits. Single-hit charge distributions during a several- $\mu \mathrm{s}$ time window for each tube are accumulated for times that precede normal data triggers by about $1 \mu \mathrm{s}$; the mean value is taken as pC per photoelectron. 


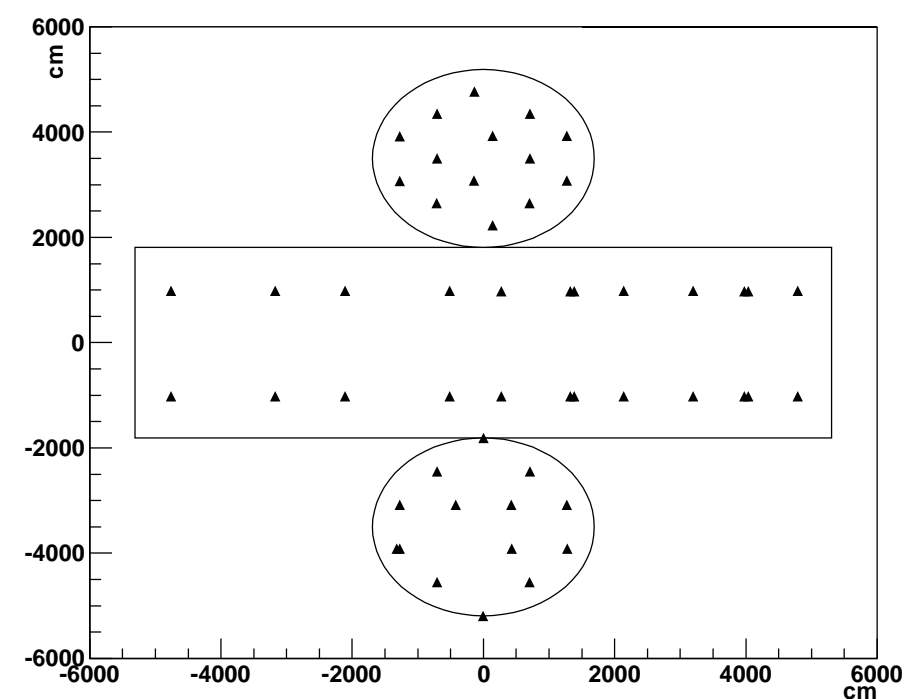

Figure 35: OD fiber end positions in "unrolled" view of the cylindrical tank. Note that some fibers occupy the same position and are overlapped in the plot. 
To confirm the charge response per photoelectron at low light levels, where the response of the OD-PMTs and electronics is linear to a good approximation, the laser was flashed at very low light levels: the occupancy $H$ (the fraction of laser pulses resulting in a hit) is related to the mean measured photoelectron value according to $\langle p e\rangle=-\ln (1-H) / H$ [12]. For data with $H<0.18$, such that $\langle p e\rangle\langle 1.1$, the mean charge value for the hit distribution was chosen to be the singlepe level. The results from the laser and dark rate methods were found to be in agreement within $10 \%$. As the dark rate method for OD charge calibration causes no detector downtime and allows for continuous monitoring, it is currently used as the primary method. For SK-IV, typical OD pC per photoelectron (pC-per-pe) values range from one to six. The $\mathrm{pC}$-per-pe values measured this way are used to determine photoelectrons for each PMT hit in the data.

Average stability of the OD charge calibration in SK-IV is shown in Fig. 36. The values of pC-per-pe are typically stable within at most $5 \%$ for a given tube over a one year period. There is a slow upward average drift as a function of time, which corresponds $\sim 1 \%$ difference on average per year. The figure also shows an increasing error on the mean gain, which implies that the PMT-to-PMT spread of gains also increases slightly with time, especially for old OD tubes. The upward drift is more pronounced for the new (R5912) PMTs than for the recycled IMB tubes. To account for the drift, new pC-per-pe values are determined on an approximately yearly timescale.

For SK-IV, only the linear charge conversion is applied to the data, i.e. the number of photoelectrons is determined for a given hit using the pC-per-pe calibration constant according to $\mathrm{pe}=\mathrm{pC} / \mathrm{pC}$-per-pe. For OD hits in SK-IV, the MC single-pe charges are chosen separately from charge distributions for old and new OD tubes, with shapes based on the average old and new tube single-pe charge distributions, respectively. In contrast to the ID case, quantum efficiencies (or light collection efficiencies) are not measured directly, but rather are treated as parameters to be tuned in SK-MC. Similarly, although charge nonlinearity properties of OD tubes at high charge were determined using the laser system (see below) and are simulated in SK-MC, nonlinearity corrections are not applied directly to the data.

\subsection{2. $O D-P M T$ nonlinearity}

The response of some typical OD-PMTs to a range of light levels injected with the OD laser system is seen in Fig. 37

Above a few hundred photoelectrons, a saturation effect appears in the measured charge. These data are fitted to

$$
Q_{\text {meas }}=Q_{\text {true }} /\left(1+k \cdot Q_{\text {true }}\right),
$$

for each PMT and implemented in the SK-IV OD detector simulation code. In Eq. (21), $Q_{\text {meas }}$, $Q_{\text {true }}$ represent measured and true charges, and $k$ is the fitting parameter. Laser light levels in this system are controlled by an attenuator. The single-pe level was determined at low occupancy so that at low light levels, the attenuator power law could be calibrated assuming a linear response of the PMTs. Using the resulting power law, $Q_{\text {true }}$ was inferred. Because some regions of the OD are less well illuminated by the laser system, a value of $k$ was estimated for each OD-PMT with cosmic-ray muons using the maximum charge per event $(\sim 1 / k)$. Values of $k$ fall within 0.001 0.003 .

\subsection{OD timing calibration}

Since OD timing information is not currently used for any event selection or reconstruction in physics data analysis, except for selection of OD hits within time windows around the trigger time, the goal of the OD timing calibration is to confirm that both the global time offset between 


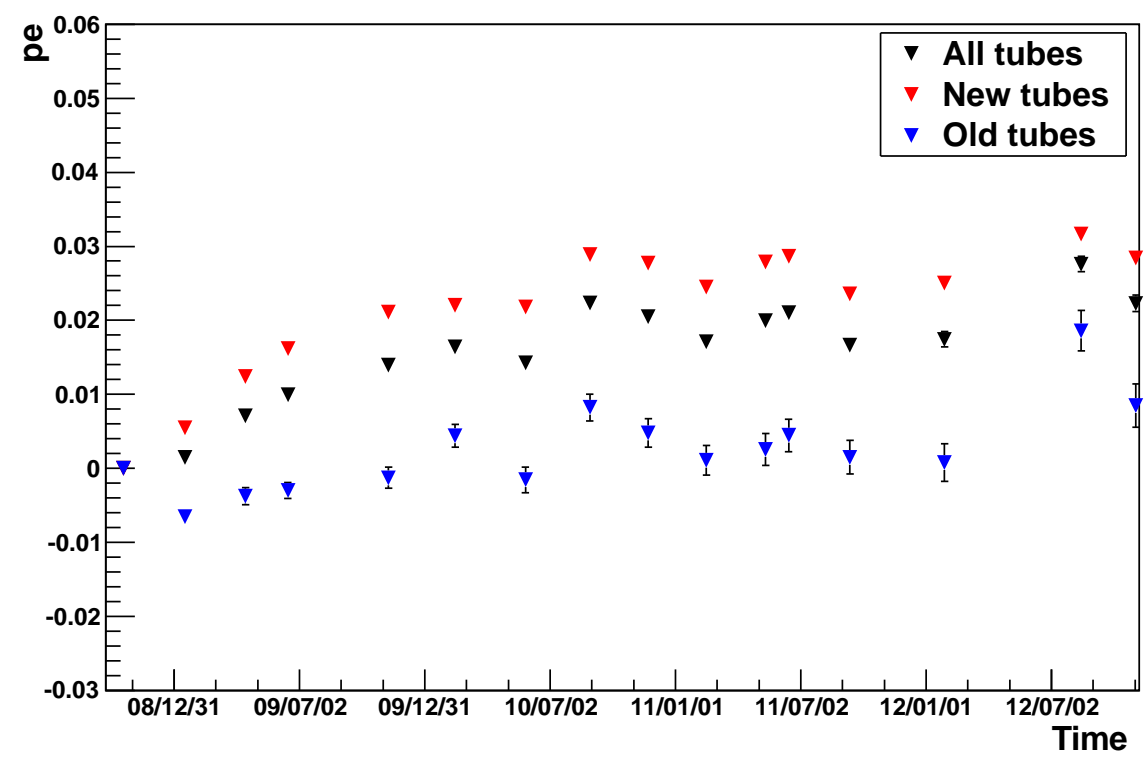

Figure 36: Average OD-PMT gain drift as a function of time, given as a fraction of a photoelectron. The plot shows the difference in pC-per-pe, averaged over PMTs, from the value for reference run near the beginning of SK-IV. The plot shows new and old tubes separately. The error bars represent the error on the mean. 


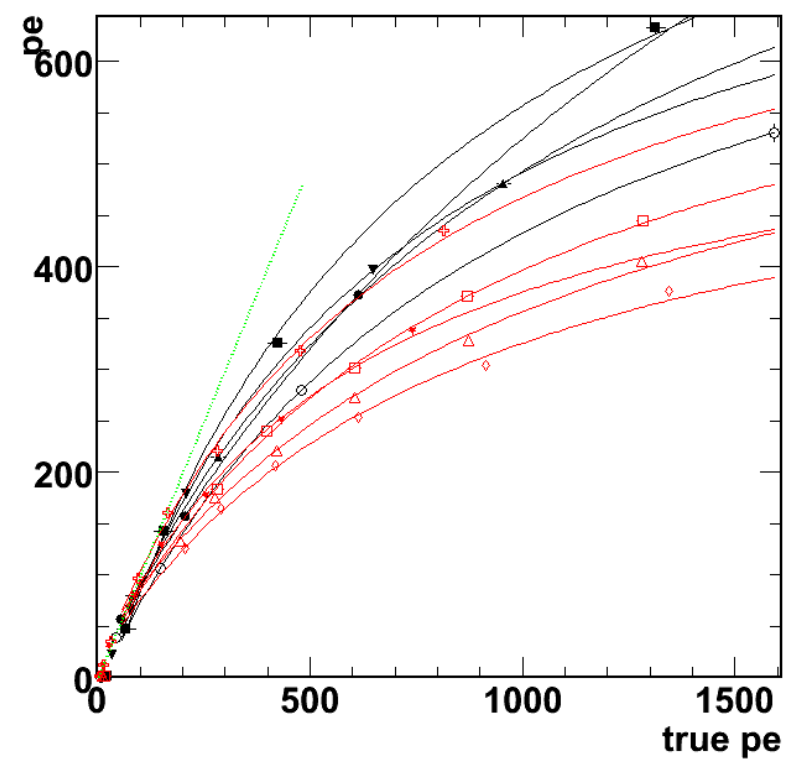

Figure 37: Correlation between input and measured charges in pe, for pe $=\mathrm{pC} / \mathrm{pC}$-per-pe, for typical old (red) and new (black) PMTs from laser data taken in March 2009 for SK-IV. Each line corresponds to a particular PMT. The result of the fit explained in the text is overlaid for each PMT. The green line is for pe $=$ true pe. 
ID and OD and the relative timing offsets for each OD-PMT are sufficiently small, say within several ns (see Section 4.1). In OD standard calibrations for the time-walk effect for OD-PMTs are not included.

For the relative timing offset, differences in cable lengths are considered. About $87 \%$ of the cables (1647 among 1885) are $70 \mathrm{~m}$ long, and the remaining have lengths between $71 \mathrm{~m}$ and $78 \mathrm{~m}$.

The global time offset was determined with the laser system, and checked with cosmic ray muons.

In October 2008, laser events were taken by flashing the laser in the ID center and the OD top. For this study, the same optical fiber and diffuser ball were used, so as not to introduce any unknown systematic differences between different installations in the ID and OD. In this way, the global OD/ID timing offset was determined to be within several ns. The relative offsets of individual PMTs were also confirmed to be within several ns.

Also in October 2008, the global time offset was independently confirmed with cosmic-ray muon data . For each event, for muon tracks fit using ID information, differences were determined between recorded times for the ID and OD-PMTs nearest to the muon track. Using the global offset parameter determined with laser data, the global timing offset was found to be within a few ns, and the OD resolution for determining the time the muon passed through the OD was found to be within about $10 \mathrm{~ns}$ (Fig. 38). As a stability check, the time offset was reconfirmed in February 2010.

\subsection{Optical properties of the $O D$}

In contrast to the ID case, the optical properties of OD materials are not measured directly, but are treated as parameters to be tuned in SK-MC. They are given nominal values and then adjusted slightly to best match quantities related to number of hits and charge for cosmic ray muons. In the MC simulation, the reflectivity of Tyvek is modeled as a combination of Gaussian specular reflection and Lambert diffuse reflection [23], with relative contributions varying as a function of angle. To obtain a starting point for this tuning, relative amounts of Gaussian and Lambertian reflection were measured ex-situ [24], and then adjusted as part of OD simulation tuning. Other tuned parameters in the simulation include relative reflectivity of Tyvek on each OD surface and transmissivity of Tyvek for the segmentation barriers. OD light collection efficiency is also tuned, as a separate average parameter for each of the three optically separated segments of the OD: top, bottom and barrel. This quantity takes into account both quantum efficiency and collection of photons.

\section{Summary}

Super-Kamiokande is a multi-purpose detector for the measurements of solar, atmospheric, and astrophysical neutrinos as well as to search for nucleon decay. It has also been used as far detector for long-baseline experiments using accelerator-produced neutrino beams. It has achieved world-leading scientific results, such as the first confirmed discovery of neutrino oscillations. To obtain such results, it is crucial to have precise detector calibrations over a very wide range, from very weak one-photoelectron light levels to quite strong, multi-hundred photoelectron light levels; these levels correspond to physics events ranging from a few $\mathrm{MeV}$ to above one $\mathrm{TeV}$. Our calibration work has been along two main lines. One is the determination of conversion factors from raw counts by the electronics to charges and times observed by PMTs. The other is modeling of optical properties of the water and detector. 

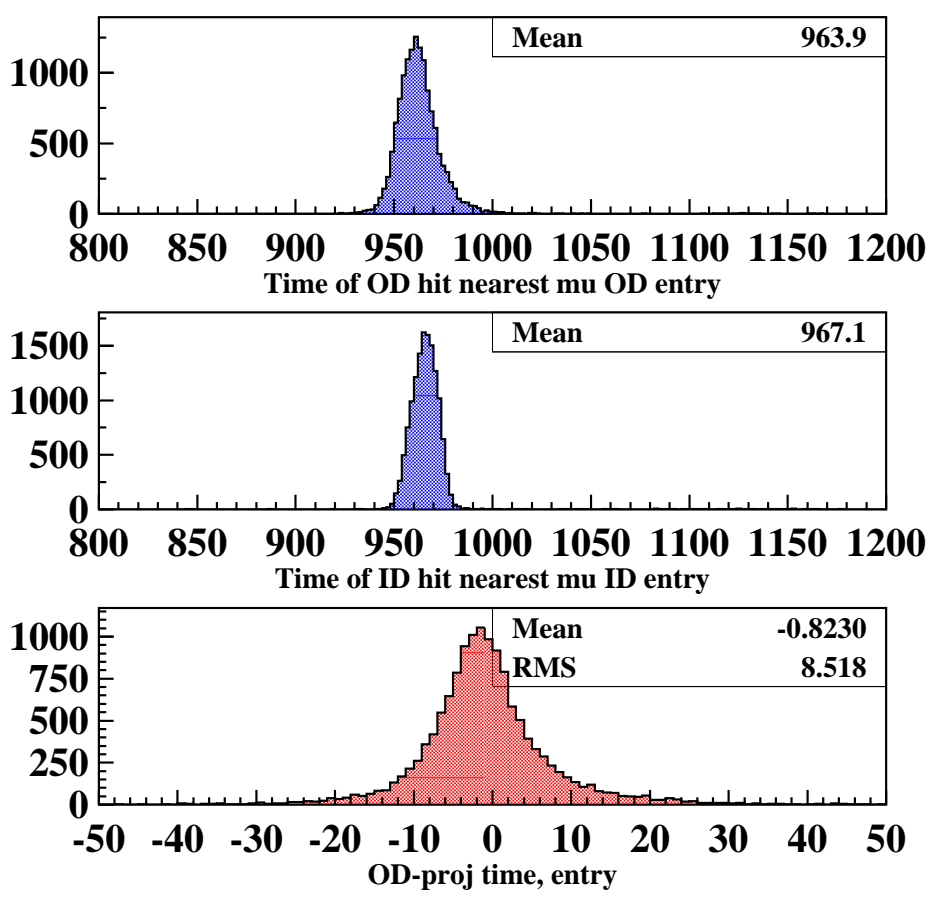

Figure 38: Top: Distribution of times (ns) for the nearest hit OD-PMT to the fit tracks of downward cosmic ray muons (arbitrary zero time value). Center: Time distribution for nearest hit ID-PMTs. Bottom: Distribution of difference between ID and OD time after correction for time of flight of the muon. 
For charge determinations, we employ a new method that allows independent determination of PMT gain and quantum efficiency. Our goal is to understand these quantities at the level of $1 \%$, and this goal has been achieved. We also observed some unexpected PMT features, such as a time dependence in quantum efficiency.

The time response of the readout channels (PMTs and readout electronics) have been calibrated for different charge ranges, covering the entire dynamic range of charge, from $10^{-1}$ to $10^{3}$ photoelectrons. The time resolution achieved by SK is, e.g. $2.1 \mathrm{~ns}$ at $\sim 1$ photoelectron and $0.5 \mathrm{~ns} \sim 100$ photoelectrons.

Optical properties of the detector were measured using several light sources. In MC simulations, we consider results of measurements of absorption and scattering probability in SK water, including its position dependence and reflections on PMT surfaces and the black sheet.

Calibrations were also performed to determine charge and timing response of the OD, as well as its optical properties. These parameters are now understood well enough to support physics analyses.

Through this calibration work, we have developed several new tools. The established calibration methods described in this paper will also be useful for future water Cherenkov detectors.

\section{Acknowledgments}

We gratefully acknowledge the cooperation of the Kamioka Mining and Smelting Company. The Super-Kamiokande experiment has been built and operated from funding by the Japanese Ministry of Education, Culture, Sports, Science and Technology, the United States Department of Energy, and the U.S. National Science Foundation. Some of us have been funded by the Korean Research Foundation (BK21), the National Research Foundation of Korea (NRF-2009-C00046), the State Committee for Scientific Research in Poland (Grant No. 1757/B/H03/2008/35), the Japan Society for the Promotion of Science, the National Natural Science Foundation of China under Grant No. 10875062, and the Spanish Ministry of Economy and Competitiveness (Grant No. FPA2009-13697-C04-02).

\section{References}

[1] Y. Fukuda et al., Nucl. Inst. and Meth. A501, 418 (2003).

[2] M. Nakahata et al., Nucl. Inst. and Meth. A421, 113 (1999).

[3] E. Blaufuss et al., Nucl. Inst. and Meth. A458, 638 (2001).

[4] J. Hosaka et al., Phys. Rev. D73, 112001 (2006).

[5] Y. Ashie et al., Phys. Rev. D71, 112005 (2005).

[6] C. Regis et al., Phys. Rev. D86, 012006 (2012).

[7] Y. Ashie et al., Phys. Rev. Lett., 93, 101801 (2004).

[8] T. Tanimori et al., IEEE Trans. Nucl. Sci., NS-36, 497 (1989).

[9] H. Nishino et al., Nucl. Inst. and Meth. A610, 710 (2009).

[10] A. Suzuki et al., Nucl. Inst. and Meth. A329, 299 (1993).

[11] R. Becker-Szendy et al., Nucl. Inst. and Meth. A324, 363 (1993).

[12] R. Becker-Szendy et al., Nucl. Inst. and Meth. A352, 629 (1995).

[13] M.E.C. Swanson et al., Astrophys. J., 652, 206 (2006).

[14] I. Thormahlen, K.Scheffler and J.Scaub, Proceedings of the 9th ICPS, 477 (1980)

[15] I. Thormahlen and J. Straub and U. Grigull, Journal of Physical and Chemical Reference Data, 14, 933 (1985).

[16] A. Baldini et al., IEEE Trans. Dielectr. Electr. Insul., 13, 547 (2006).

[17] E.Pope, R.Fry, Appl. Opt. 33, 8710 (1997).

[18] V. S. Langford, A. J. McKinley, and T. I. Quckenden, J. Phys. Chem. A, 105, 8916 (2001).

[19] S. Yamada et al., IEEE Transactions on Nuclear Science, 57, 428 (2009). 
[20] D. Motta and S. Schonert, Nucl. Inst. and Meth. A539, 217 (2005).

[21] K. Abe et al., Nucl. Inst. and Meth. A659, 106 (2011).

[22] Private communication with Hamamatsu K.K.

[23] A. Filevich et al., Nucl. Inst. and Meth. A423, 108 (1999).

[24] A. Chavarria, A study on the reflective properties of Tyvek in air and underwater, http://phy.duke.edu/ schol/superk/alvaro_thesis.pdf' ' (2007) 